\title{
Comparative analysis of cyanobacteria species reveals a novel guanidine-degrading enzyme that controls genomic stability of ethylene-producing strains
}

\section{Bo Wang ( $\sim$ bo.wang.2@vanderbilt.edu )}

Vanderbilt University https://orcid.org/0000-0002-6047-1221

\section{Yao Xu}

Vanderbilt University

\section{Xin Wang}

Miami University https://orcid.org/0000-0002-7174-3042

Joshua Yuan

Texas A\&M University

\section{Carl Johnson}

Vanderbilt University

\section{Jamey Young}

Vanderbilt Univerisity https://orcid.org/0000-0002-0871-1494

Jianping Yu

National Renewable Energy Laboratory https://orcid.org/0000-0003-0466-3197

\section{Article}

Keywords: guanidine metabolism, degradation pathways, ethylene bioproduction, guanidine-degrading enzyme

Posted Date: March 29th, 2021

DOI: https://doi.org/10.21203/rs.3.rs-197190/v1

License: (1) This work is licensed under a Creative Commons Attribution 4.0 International License. Read Full License

Version of Record: A version of this preprint was published at Nature Communications on August 26th, 2021. See the published version at https://doi.org/10.1038/s41467-021-25369-x. 


\section{Abstract}

Recent studies have revealed the prevalence and biological significance of guanidine metabolism in nature. However, the metabolic pathways used by microbes to degrade guanidine or mitigate its toxicity have not been widely studied. Here we report a novel guanidine-degrading enzyme, Sll1077, identified in the model cyanobacterium Synechocystis sp. PCC 6803 through comparative proteomics and subsequent experimental validation. Although previously annotated as an agmatinase enzyme, Sll1077 is more likely a "guanidinase", because it degrades guanidine rather than agmatine to urea. We demonstrate that the model cyanobacterium Synechococcus elongatus PCC 7942 strain engineered to express the bacterial ethyleneforming enzyme (EFE) exhibits unstable ethylene production due to toxicity and genomic instability induced by accumulation of the EFE-byproduct guanidine. Co-expression of EFE and SII1077 significantly enhanced genomic stability and enabled the resulting strain to achieve sustained high-level ethylene production. These findings expand our knowledge of natural guanidine degradation pathways and demonstrate their biotechnological application to support ethylene bioproduction.

\section{Introduction}

Despite the practical applications of guanidine as a protein denaturant (when applied at high concentrations) ${ }^{1}$ and as an ingredient in slow-release fertilizers ${ }^{2}$, little is known about the fate of guanidine in biological systems. Guanidine has been detected in human urine at concentrations of $7-13 \mathrm{mg} \mathrm{L}^{-1}$ (0.12-0.22 mM $)^{3}$, but its biosynthetic pathway remains elusive ${ }^{4}$. A recent study also revealed that a variety of microorganisms, including E. coli, produce guanidine through unknown mechanisms under nutrient-poor growth conditions, suggesting that guanidine metabolism is biologically significant and is prevalent in natural environments ${ }^{5}$.

While nonenzymatic decomposition of guanidine under physiological conditions is extremely slow ${ }^{6}$, soil microbes are able to degrade guanidine using heretofore unknown metabolic pathways ${ }^{7}$. Recently, it was reported that a wide range of microorganisms possess a class of guanidine riboswitches that control the expression of downstream genes, a majority of which encode proteins involved in nitrogen metabolism, nitrate/sulfate/bicarbonate transporters, and small multidrug resistance (SMR) transporters ${ }^{5,8-11}$. The SMR transporters were found to be responsible for exporting guanidine out of cells $s^{5,12}$. A previously annotated "urea carboxylase" was reported to carboxylate guanidine to form carboxyguanidine ${ }^{5}$, which is degraded by a carboxyguanidine deiminase followed by further degradation by allophanate hydrolase ${ }^{13}$. Another class of enzymes regulated by guanidine riboswitches are annotated as "agmatinases" in the arginase superfamily $5,9,14,15$, which catalyze the breaking of C-N bonds in the guanidyl moiety of agmatine, releasing urea ${ }^{16}$. There is no current explanation for why these enzymes evolved regulation in response to free guanidine.

To date, the only known enzyme that produces guanidine is the ethylene-forming enzyme (EFE) that catalyzes formation of ethylene and guanidine simultaneously from a-ketoglutarate (AKG) and arginine ${ }^{17}$. Due to biotechnological interests in developing an alternative pathway for renewable production of ethylene, which is the most highly produced organic compound in the petro-chemical industry, the efe gene from 
Pseudomonas syringae (a plant pathogen) has been introduced into a variety of microbial species ${ }^{17}$. Some hosts, e.g., Pseudomonas putida KT2440 and the cyanobacterium Synechocystis sp. PCC 6803 (hereafter Synechocystis 6803), have been able to accommodate stable, high-level expression of EFE and thereby sustain enhanced production of ethylene ${ }^{17-22}$. Other species, such as cyanobacterium Synechococcus elongatus PCC 7942 (hereafter Synechococcus 7942) and Synechococcus elongatus PCC 11801 (hereafter Synechococcus 11801), however, have not been able to tolerate high-level expression of EFE, and the recombinant strains suffered severe growth inhibition ${ }^{23-25}$ that was rescued by spontaneous chromosomal mutations that abolished the expression of functional $\mathrm{EFE}^{23,24}$.

In this study, we report a novel guanidine-degrading enzyme discovered through comparative analysis of multiple cyanobacterial species. We show that guanidine possesses significant toxicity to cyanobacterial cells and destabilizes their genome in response to recombinant EFE expression. Synechocystis 6803 is able

to degrade and utilize guanidine as a nitrogen source through the activity of an enzyme encoded by the gene s/11077, which was previously annotated as an agmatinase in the arginase superfamily. We posit that Sll1077 is more likely a "guanidinase", because it degrades guanidine rather than agmatine to urea. This result is consistent with the finding that there is a conserved sequence motif of the guanidine riboswitch upstream of the s/l1077 ORF in the genome of the wild type Synechocystis 6803 strain. Synechococcus 7942 lacks a homologous enzyme in its genome and is unable to mitigate guanidine toxicity. We find that heterologous expression of Sll1077 in a recombinant Synechococcus 7942 strain confers the ability to degrade guanidine into non-toxic urea. Co-expression of SIl1077 and EFE in Synechococcus 7942 stabilizes the genome of the resultant strain and leads to sustained production of ethylene from light and $\mathrm{CO}_{2}$.

\section{Results}

Varied guanidine degradation capabilities are present in different cyanobacterial species. Given that the impacts of guanidine on microorganisms are unclear, we studied guanidine degradability and toxicity in two model cyanobacterial species: Synechocystis 6803 and Synechococcus 7942. In our preliminary experiments with Synechocystis 6803, when nitrate was gradually replaced with guanidine in the culture medium, the guanidine concentrations declined over a period of four days in all cases under photoautotrophic cultivation conditions (Fig. S1). In order to rule out the possibility of photochemical degradation, Synechocystis 6803 cells were resuspended in the nitrate-deprived mBG11 medium with or without $5 \mathrm{mM}$ guanidine (detailed in Materials and Methods). In parallel, Synechococcus 7942 and heatkilled Synechocystis 6803 cells were also resuspended in the nitrate-deprived culture medium supplemented with $5 \mathrm{mM}$ guanidine. We found that while Synechocystis 6803 cells grown in nitrate-deprived medium exhibited an expected chlorosis phenotype and were still able to double the amount of biomass, cells grown in the guanidine-supplemented medium were able to maintain their green pigmentation and reached a higher cell density after 6 days of photoautotrophic cultivation (Fig. 1a,b). Noticeably, the Synechocystis 6803 cells exposed to exogenous guanidine had a slower growth rate than those not exposed to guanidine during the first day, probably due to the toxicity of guanidine (Fig. 1a,b). By contrast, the cultures inoculated with heatkilled Synechocystis 6803 or live Synechococcus 7942 cells did not show a typical chlorotic phenotype, and showed continuous decline of biomass over the period of 6 days (Fig. 1a,b). While the guanidine content in 
the culture with Synechococcus 7942 or heat-killed Synechocystis cells did not decline, the continuous increase of biomass in the culture of live Synechocystis 6803 cells coincided with a steady decrease of the guanidine concentration in the culture medium (Fig. 1C). To this end, we hypothesized that a guanidinedegrading metabolic pathway may exist in Synechocystis 6803 but not in Synechococcus 7942.

Sll1077 is responsible for guanidine degradation in Synechocystis 6803 . A comparative proteomic study of the wild-type Synechocystis 6803 and the guanidine-producing (efe-expressing) strain, JU54726, showed that the expression of SII1077, a putative agmatinase, increased by 10-fold in strain JU547 compared to that in the wild-type Synechocystis 6803 (Table S1). Agmatinase cleaves the C-N bond within the guanidyl moiety of agmatine, which releases putrescine and urea ${ }^{27}$. Interestingly, expression of $s / 1077$ is predicted to be under the control of a guanidine riboswitch based on analysis of the RNA sequence upstream of its ORF (Fig. 2a, b) ${ }^{5}$. We hypothesized that SII1077 might be involved in the metabolism of guanidine in Synechocystis 6803 (Fig. 2c). Knockout of s/l1077 in Synechocystis 6803, leading to strain PB805W $(\Delta s / / 1077)$, did not have any apparent physiological effects on the cells under normal growth conditions (data not shown), or under nitrate-deprived conditions (Fig. 2d-e). Nevertheless, under nitrogen-deprived guanidine-supplemented culture conditions, the cell growth of $\Delta$ s/l1077 was severely inhibited compared to the wild-type Synechocystis 6803 and the degradation of the light harvesting components, i.e., phycobilisomes (absorbance at $630 \mathrm{~nm}$ ) and chlorophyll a (absorbance at $680 \mathrm{~nm}$ ), in $\Delta s / 11077$ was remarkably retarded compared to the wild-type Synechocystis 6803 or $\Delta$ s//1077 cultivated in nitrogendeprived medium (Fig. 2d-f). Further analysis revealed that the guanidine degradation capability was abolished in the Synechocystis $\Delta$ s/l1077 strain (Fig. 2g), a phenotype similar to that of wild-type Synechococcus 7942 (Fig. 1a). In addition, during the first day, the biomass of strain $\Delta$ s/l1077 incubated with guanidine increased to a much less extent relative to other parallel cases; in the next few days, the biomass of strain $\Delta s / 1077$ incubated with guanidine underwent an autolysis process and the light harvesting complex gradually deteriorated (Fig. 2d-f).

Overexpression of s/1077 in Synechocystis 6803 was achieved through optimizing the ribosome binding site (RBS) at the $5^{\prime}$ region as well as tailoring the $3^{\prime}$ region of the expression cassette (Fig. 3a). Among the six tested RBSs, RBSv309 in strain PB809W rendered the strongest expression level (Fig. 3b). While removal of the Xhol restriction site between the s/l1077 and the $6 \times$ His tag sequence at the 3 ' region in strain PB812W did not have any apparent effect on the s/l1077 expression level, adding the rnB T1T2 terminator (from E. coli) to the 3' region significantly improved the expression of sl/1077 in PB816W (Fig. 3a,b). Strain PB816W was able to degrade guanidine at a rate approximately $80 \%$ faster than the wild-type Synechocystis 6803 , which led to a faster cell growth rate in nitrate-deprived medium (Fig. 3c,d). Interestingly, although removal of the $6 \times$ His tag sequence from the 3 ' end of s/11077 did not affect the protein expression level (Fig. 3b), it increased the guanidine degradation rate by about two times and substantially increased the cell growth rate of PB817W (Fig. 3c,d), suggesting that the C-terminus 6 x His tag negatively impairs the guanidine-degrading enzyme activity of Sll1077.

In order to verify that guanidine is degraded by SII1077 to form urea, according to the enzymatic mechanism of the agmatinase/arginase superfamily ${ }^{27}$, SIl1077-His was purified from the crude cell lysate of Synechocystis strain PB816W (Fig. 3b). Purified SII1077-His showed an apparent molecular weight of $\sim 45$ 
$\mathrm{kDa}$ which is consistent with the predicted molecular weight of $43.8 \mathrm{kDa}$ (Fig. 4a). Incubation of purified Sll1077-His with guanidine at $30^{\circ} \mathrm{C}$ resulted in hydrolysis of guanidine and release of urea (Fig. $4 \mathrm{~b}-\mathrm{d}$ ). It is noteworthy that no reducing factors, such as ATP or $\mathrm{NAD}(\mathrm{P}) \mathrm{H}$, are required to drive the guanidine hydrolysis enzymatic activity of SII1077, which seems to be more energy-efficient compared to the previously reported guanidine carboxylation pathway ${ }^{5,13}$ (Fig. 4e).

Expression of sll1077 improves tolerance of Synechococcus 7942 to guanidine. In order to examine if expressing a recombinant enzyme, SII1077 from Synechocystis 6803, could endow the guanidine degradation capability in a host strain that does not naturally degrade guanidine, we expressed s/17077 in Synechococcus 7942, resulting in strain GD7942 (+ s//1077). While the cell growth of Synechococcus 7942 was already inhibited by guanidine at concentrations as low as $0.3 \mathrm{mM}$ and was severely inhibited by $1 \mathrm{mM}$ guanidine under photoautotrophic conditions (Fig. 5a), the sll1077-expressing strain GD7942 gained significant tolerance to exogenous guanidine. Particularly, the cell growth of strain GD7942 was not apparently repressed by as much as $1 \mathrm{mM}$ guanidine present in the culture medium, and was only slightly inhibited by $2 \mathrm{mM}$ guanidine (Fig. 5a). We further examined the fate of the exogenous guanidine in the culture medium containing $1 \mathrm{mM}$ guanidine. As expected, while no guanidine degradation occurred in the culture of wild-type Synechococcus 7942, the guanidine added into the culture medium of the GD7942 strain was completely degraded over 4 days of photoautotrophic cultivation (Fig. 5b). Since the wild-type Synechococcus 7942 does not have any urea biosynthesis or degradation pathways ${ }^{28}$, it was expected that urea would be accumulated in the GD7942 culture. Indeed, along with the degradation of guanidine, urea gradually accumulated in the culture supernatants to concentrations of about $1 \mathrm{mM}$ by end of day 4 (Fig. 5b), which is consistent with the pathway annotation ${ }^{28}$ and enzymatic reaction stoichiometry (Fig. 2a). We further found that supplementing $5 \mathrm{mM}$ urea into the culture medium of Synechococcus 7942 did not show any apparent impact on the cell growth under either nitrate-deprived or nitrate-replete culture conditions (Fig. S2), suggesting that Synechococcus 7942 is highly tolerant to urea.

SIl1077 prefers guanidine rather than agmatine as the substrate. To examine the substrate preference of Sll1077 towards guanidine and agmatine, the crude cell extract of Synechococcus 7942 and GD7942 was incubated with $5 \mathrm{mM}$ of either guanidine or agmatine at $30^{\circ} \mathrm{C}$. Surprisingly, we found that the crude cell extract of GD7942 was able to degrade guanidine but not agmatine. The concentration of guanidine incubated with the GD7942 cell lysate decreased by about $2 \mathrm{mM}$, and concomitantly about $2 \mathrm{mM}$ urea was produced in the reaction mix over the examined $12 \mathrm{~h}$ time period (Fig. $5 \mathrm{c}, \mathrm{d})$. We therefore propose denominating Sll1077 as a "guanidinase" instead of an agmatinase.

\section{Co-expression of SII1077 and EFE enhances genomic stability and sustains high-level ethylene formation in} Synechococcus 7942. Given that the EFE reaction produces not only ethylene but also toxic guanidine, which might be responsible for the genomic instability observed upon expression of EFE alone in Synechococcus $7942^{23,24}$, we examined if co-expressing SIl1077 and EFE in the Synechococcus 7942 host strain would render a stable genome and thereby sustained production of ethylene. We found that following the genetic transformation of Synechococcus 7942 and colony-restreaking on BG11 agar plates, the recombinant efe-expressing strain, EFE7942, grew considerably slower than wild-type and the initially formed colonies appeared yellow-greenish; subsequently, large and dark-green colonies grew up on the 
background of the smaller colonies (Fig. 6a). Cultivation of these "large" and "small" colonies in the liquid culture revealed that cells from the small colonies, but not from the large ones, retained photosynthetic ethylene productivity. Subsequent colony PCR and DNA sequencing results confirmed that cells from the small colonies retained the correct EFE expression cassette on their genomes, whereas the large colonies consisted of cells with mutations around the EFE expression cassette which abolished expression of EFE (Fig. S3). It is noteworthy that restreaking single small colonies onto fresh mBG11-agar plates supplemented with spectinomycin repeatedly resulted in a mixture of large and small colonies after 1-2 weeks of incubation at $30^{\circ} \mathrm{C}$, indicating a constant selective pressure caused by the expression of EFE. In contrast, co-expression of Sll1077 with EFE in Synechococcus strain GD-EFE7942 resulted in uniform colony sizes on agar plates at $30^{\circ} \mathrm{C}$ (Fig. 6a), and colony PCR and DNA sequencing confirmed that these cells were able to maintain the intact EFE expression cassette on their genome (Fig. S5), indicating relief of the selective pressure caused by the expression of EFE. Because EFE exhibits highest enzyme activity in the temperature range of $20-25^{\circ} \mathrm{C}$ and becomes unstable at temperature above $30^{\circ} \mathrm{C} 29,30$, we decided to routinely maintain strain EFE7942 at $35^{\circ} \mathrm{C}$ to suppress the EFE activity and thereby prevent spontaneous mutations from occurring.

The wild-type Synechococcus 7942 strain and the efe-expressing strains EFE7942 and GD-EFE7942 were then compared in regard to their cell growth rates and ethylene productivities in liquid cultures at $30^{\circ} \mathrm{C}$ under photoautotrophic culture conditions. Initially, strain EFE7942 grew considerably slower than the wild-type Synechococcus 7942 strain, but gradually grew faster after subsequent re-inoculations, reaching a growth rate similar to that of the wild-type by day 13 . In contrast, the GD-EFE7942 strain exhibited a slightly slower growth rate compared to the wild-type strain throughout the entire 13-day cultivation period (Fig. 6b). In terms of the ethylene production, during the first 9 days strain GD-EFE7942 showed 3- 6 times higher volumetric ethylene productivities compared to strain EFE7942, with more substantial differences occurring at relatively high cell densities when guanidine accumulated to the highest levels in the culture medium (Fig. 6c, S4). The higher volumetric ethylene productivity of GD-EFE7942 relative to EFE7942 was largely due to the improved cell growth rate and thereby higher cell density (Fig. 6b), yet was also attributed to the improved specific ethylene productivity (Fig. 6d). During the first 7 days, the specific ethylene productivity of GD-EFE7942 was 1.2-1.8 times higher than EFE7942. The difference increased to 2.6 times by day 8 , and to 3.3 times by day 9 (Fig. 6 d). Starting from day 10, both the volumetric and specific ethylene productivities of strain EFE7942 dropped substantially and declined to almost zero by day 13 (Fig. 6c,d). The guanidine production in the EFE7942 culture also started to drop significantly by day 10 (Fig. S4). Absorbance spectra of the three examined cultures revealed that the abundance of phycobilisome and chlorophyll a in EFE7942 declined significantly compared to those in the wild-type strain. Although the phycobilisome level remained low in GD-EFE7942 relative to that of the wild-type strain, expression of SII1077 restored the amount of chlorophyll a in GD-EFE7942 to a level similar to that in the wild-type strain (Fig. 6e). Further cell growth phenotyping and DNA sequencing analyses revealed that after 13 days of cultivation, approximately half the cells in the EFE7942 culture lost the entire EFE expression cassette, and the other half had DNA mutations on the genome that caused early termination of translation of EFE (Fig. S5, 6f-g). By contrast, the GDEFE7942 strain exhibited consistent cell growth profiles and ethylene productivities during the five consecutive batch cultures (Fig. 6b-d), owing to its engineered capability to mitigate guanidine via SII1077 
(Fig. 5b,S4). In addition, the ethylene productivity of GD-EFE7942 is comparable to that of the previously engineered high-level-efe-expressing Synechocystis strains, e.g., strain PB752 in our previous work ${ }^{31}$, under the examined photoautotrophic culture conditions (Fig. S6).

\section{Discussion}

Through comparative analysis of cyanobacterial strains, we were able to identify a novel guanidinedegrading enzyme, SII1077, which breaks down guanidine to form urea and ammonium (Fig. 2a, 4, 5b,c). SIl1077 constitutes a guanidine degradation pathway that does not require ATP, and is completely different from the recently identified guanidine carboxylation pathway ${ }^{5,13}$ (Fig. 4e). Guanidine carboxylase catalyzes the carboxylation of guanidine using ATP as the driving force. However, the product compound carboxyguanidine is unstable and is readily hydrolyzed to form guanidine and $\mathrm{CO}_{2}$ in water, forming an ATPconsuming futile cycle ${ }^{13}$. The efficiency of the guanidine carboxylation pathway largely depends on the rate of removal of carboxyguanidine by the carboxyguanidine deiminase which converts carboxyguanidine to ammonium and allophanate ${ }^{13}$. In contrast, the guanidine-degrading enzyme SII1077 investigated in the current study acts as a deiminase and is able to, without consuming ATP, directly convert guanidine to urea which could be further degraded into $\mathrm{CO}_{2}$ and ammonium by the urease in most cyanobacterial species, including Synechocystis $6803^{28}$ (Fig. 4). Therefore, the SII1077-associated guanidine degradation pathway seems more energy-efficient compared to the guanidine carboxylation pathway.

SIl1077 represents a class of novel guanidine-degrading (i.e., "guanidinase") enzymes. Both SIl1077 and SIl0228 in Synechocystis 6803 have been annotated as putative agmatinases since they both have the conserved regions of the agmatinase/arginase superfamily proteins ${ }^{15,32}$. However, their protein sequences show less than 25\% identities (Fig. S7). A previous study reported that neither $s / 1077$ nor s/l0228 contributes to the arginase activity, while the agmatinase activity in Synechocystis 6803 is mostly attributed to $s / 10228^{33}$. A recent study also showed that deletion of $s / 10228$ rather than $s / 1077$ significantly impairs the utilization of arginine in Synechocystis $6803^{34}$. Additionally, from a bioinformatics approach it was found that the expression of s/11077 and its analogs (previously annotated to encode

"agmatinase"/"arginase" enzymes) in a wide range of microorganisms is under the control of guanidine

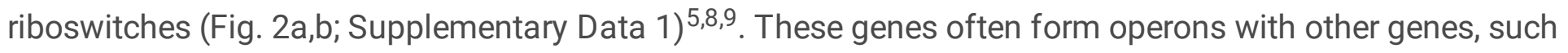
as hypA, hypB, SsuA_fam (s/l1080 in Synechocystis 6803), TM_PBP2 (s/l1081 in Synechocystis 6803) and ABC_NrtD_SsuB (s/l1082 in Synechocystis 6803) (Supplementary Data 1) ${ }^{5}$. The expression levels of these genes were all enhanced in guanidine-producing Synechocystis strains compared to wild-type controls according to our proteomic data (Table S1) and results from a previous transcriptomic study ${ }^{35}$, which is consistent with the modulation mechanism of guanidine riboswitches ${ }^{5}$. Taken together, these results suggest that SII1077 may be evolved for a function that is completely different from the degradation of arginine or agmatine. Our findings that SII1077 is able to degrade guanidine and that it prefers guanidine rather than agmatine as substrate is consistent with the prediction that its expression is under the control of a guanidine riboswitch (Fig. 2a,b) ${ }^{5}$, which suggests that SII1077 and possibly its analogs are evolved for the degradation of guanidine. It is likely that guanidine, formed either biologically or abiotically, is present in the 
natural environment where Synechocystis 6803 lives, and possessing s/l1077 has rendered survival advantage. Running a protein BLAST for the Sll1077 peptide sequence (https://blast.ncbi.nlm.nih.gov/) returned over a thousand hits with $>50 \%$ sequence identities, all of which have been annotated as arginase/agmatinase family proteins (Supplementary Data 2). Whether these proteins possess the capability to degrade guanidine needs to be studied in the future.

Guanidine causes a disorder of pigment metabolism in cyanobacterial cells. Guanidine is known to interact with the peptide backbone and side-chains of amino acids, and serves as a protein denaturant when applied at high concentrations $(2-6 \mathrm{M})^{1,36,37}$. At concentrations insufficient to completely unravel the protein structure, guanidine could also be detrimental to biomacromolecules. For example, relatively small amounts of guanidine could trigger unfolding of the active site of ribonuclease $A$ and thereby inactivate the enzyme activity and facilitate the proteolysis process ${ }^{38}$. Another example is that millimolar guanidine could significantly inhibit ammonium nitrification in the nitrifying bacteria in soil ${ }^{39}$. In our study, the presence of guanidine in the culture medium, either from exogenous or endogenous sources, severely inhibited cell growth of wild-type Synechococcus 7942 and the Synechocystis $\Delta$ s/l1077 strain (Fig. 1a,b, 2d,e, 5a, 6a,b). These guanidine-sensitive strains exhibited remarkably slow degradation of their light harvesting components under nitrate-deprived and guanidine-supplemented culture conditions (Fig. 1a, 2e,f). Under nitrogen-poor culture conditions, cyanobacterial cells typically undergo a chlorosis process that involves degrading their phycobiliproteins and chlorophyll as a nitrogen source to support cell growth while simultaneously downregulating photosynthesis in order to reduce the generation of damaging oxygen radicals ${ }^{40}$. Impaired cell growth and retarded pigment degradation in both cultures of Synechocystis $6803+$ and $\Delta s / / 1077+$ on day 1 (Fig. $2 \mathrm{~d}$-f) suggested that induction of nitrogen chlorosis was disrupted by guanidine under the examined culture conditions. Furthermore, the biosynthesis of phycobiliproteins and chlorophyll was severely inhibited in strain Synechococcus EFE7942, whereas the biosynthesis of chlorophyll was restored through heterologous expression of SII1077 in strain GD-EFE7942 (Fig. 6e), which provided additional evidence that guanidine hampers the biosynthesis and remodeling of photosynthesisrelated pigments in cyanobacteria.

While the wild-type Synechococcus 7942 is sensitive to guanidine and fails to accommodate high-level expression of EFE (Fig. 1a,b, 5a, 6), our discovery of the guanidine-degrading activity of Sll1077 was leveraged to generate a derivative strain of Synechococcus 7942 that exhibits enhanced genomic stability and stable high-level production of ethylene in prolonged culture, which has not been achieved in prior studies (Fig. 6, S5) $23-25,41$. It is noteworthy that co-expression of SII1077 with EFE substantially attenuate, but does not completely eliminate, the accumulation of guanidine in cultures of the engineered Synechococcus GD-EFE7942 strain (Fig. S4). Although this seems already sufficient for rendering genomic stability and sustained stable ethylene production in GD-EFE7942 (Fig. 6, S5), as well as the Synechocystis strain $\mathrm{PB} 752^{26}$, it could be possible to obtain a more efficient guanidine-degrading enzyme, perhaps through directed evolution of SII1077, in order to achieve faster degradation of guanidine and further reduce its toxicity in the future. In summary, this study has advanced our understanding of the biological routes of guanidine metabolism in nature and has demonstrated a new approach for enhancing biosynthesis of target 
molecule(s) by reducing toxic byproduct(s), focusing upon the specific example of stabilizing ethylene production in engineered microorganisms.

\section{Materials And Methods}

Bacterial strains and growth conditions. E. coli NEB5a (New England BioLabs, MA, USA) served as the microbial host for cloning and maintaining all recombinant plasmids, and was routinely grown in LB medium. Synechocystis and Synechococcus strains were typically grown in a modified BG11 medium (mBG11) as described before ${ }^{26}$, and $N$-tris(hydroxymethyl)methyl-2-aminoethanesulfonic acid (TES) and $\mathrm{NaHCO}_{3}$ were supplemented to final concentrations of $20 \mathrm{mM}$ and $100 \mathrm{mM}$, respectively, unless otherwise specified. The medium was filtered through sterile $0.22 \mu \mathrm{m}$ membranes before use. Cyanobacterial liquid cultures were grown under constant light of about $50 \mu \mathrm{E} \mathrm{m}^{-2} \mathrm{~s}^{-1}$ on a rotary shaker at $150 \mathrm{rpm}$ and $30^{\circ} \mathrm{C}$ in a Percival chamber (Percival Scientific, Inc., IA, USA) aerated with $5 \% \mathrm{CO}_{2}$ unless otherwise specified. When cyanobacteria were grown on solid medium, $10 \mathrm{mM}$ TES, $3 \mathrm{~g} \mathrm{~L}^{-1}$ thiosulfate and $15 \mathrm{~g} \mathrm{~L}^{-1}$ agar were supplemented to the $\mathrm{mBG} 11$ medium, and sterilized by autoclaving at $121^{\circ} \mathrm{C}$ for $30 \mathrm{~min}$. When appropriate, antibiotics were added to the solid medium to the following final concentrations: $50 \mathrm{mg} \mathrm{L}^{-1}$ for spectinomycin and $7 \mathrm{mg} \mathrm{L}^{-1}$ for chloramphenicol, respectively. Synechococcus sp. PCC 7002 was grown in $\mathrm{A}^{+}$medium for general maintenance purpose. All strains and plasmids used in this study are listed in Table S2.

Construction of recombinant plasmids. All enzymes and cloning kits were purchased from New England Biolabs, MA, USA, unless otherwise specified. Kits for DNA purification were purchased from Qiagen, MD, USA. Plasmid pPB305 was constructed by PCR amplification of the DNA fragments of sll1077U, sll1077D, and cat, and Gibson Assembly into plasmid pBlueScript II SK (+) which was digested with Kpnl and Sacl. The DNA fragment containing gene s/l1077 was PCR amplified from the genomic DNA of Synechocystis 6803 and inserted between the Ndel and Xhol restriction sites on pET30a(+), so that Sll1077 will be tagged with 6xHis, resulting in plasmid pPB300. pPB306 was constructed by PCR amplifying s/17077-His from pPB300 and inserting it between the Ndel and Sall restriction sites on pSCPTH (Wang, 2013) using Gibson Assembly Kit. pPB306d was constructed by deleting the lac promoter region on the pBluescript vector backbone via digesting pPB306 with Sacl and Sapl restriction enzymes and then blunt-ended using T4 DNA polymerase and self-ligated using Quick DNA ligase. pPB307, pPB308, pPB309, pPB310, pPB311 were constructed by replacing the RBS in pPB306d using the Site Directed Mutagenesis Kit. pPB312 was constructed by deleting the "CTCGAG" (Xhol) nucleotides between the sll1077 coding sequence and the 6xHis tag on plasmid pPB309. pPB316 was constructed by inserting the rrnBT1T2 terminator (from E. coli NEB5a) downstream of s/l1077 on pPB312. pPB312 was digested with Sall, dephosphorylated and then assembled with the terminator rrnBT1T2 using Gibson Assembly Kit. pPB313 was constructed by deleting the 6xHis tag and "CTCGAG" (Xhol) between the sll1077 coding sequence and stop codon TAA of pPB309. pPB317 was constructed by inserting rrnBT1T2 downstream of s/l1077 on pPB313, which was digested with Sall and dephosphorylated, using the Gibson Assembly Kit. Plasmid To express the efe gene in Synechococcus 7942, $1.43 \mathrm{~kb}$ of the $\mathrm{BbvCl} / \mathrm{Xhol}$ fragment containing $p s b A p$ ::efeFLAG from pJU158 was blunt-ended and ligated to the Smal site of the neutral site 1 vector pAM1303, resulting in PEFE-FLAG-NS1. 
To overexpress the s/l1077 gene in Synechococcus 7942, $1.69 \mathrm{~kb}$ of the BamHI/Sall fragment harboring the s/11077 expression cassette from pPB317 was cloned into the BamHI/Sall site of a neutral site 4-targeting vector pCX0104-LuXAB-FT ${ }^{42}$ to generate pGD7942-NS4. The DNA sequence of genes of interest were all conformed by DNA sequencing. Primers used in constructing all plasmids are detailed in Table S2.

Genome engineering of cyanobacteria. Transformation of Synechocystis was accomplished via natural transformation as described previously ${ }^{43}$. Briefly, the wild-type Synechocystis 6803 strain was grown in mBG11 medium until the $\mathrm{OD}_{730}$ reached approximately 0.4 . Then, $2.5 \mathrm{~mL}$ of culture was condensed to about $0.2 \mathrm{~mL}$ via centrifugation and resuspension with the same culture medium. Cells were transferred into a 1.5 $\mathrm{mL}$ Eppendorf tube and mixed with 1-2 $\mu \mathrm{g}$ DNA of integration plasmid. The sample was incubated under low light for about 5 hours, and mixed once in the middle of the incubation. Cells were then spread onto BG11 plates supplemented with appropriate antibiotics. Strains PB805W - PB812W, PB816W, PB817W were constructed by transforming wild-type Synechocystis 6803 with integration plasmids pPB305 - pPB312, pPB316 and pPB317. Strains PB816H and PB817H were constructed by transforming an efeexpressing strain, Synechocystis PB752, with the integration plasmids pPB316 and pPB317, respectively.

Transformation of Synechococcus 7942 was completed following a previously established protocol ${ }^{44}$. Transformation of Synechococcus 7942 with integration plasmids pEFE-FLAG-NS1 or pGD7942-NS4 resulted in strain EFE7942 and GD7942, respectively. The efe expression cassette was PCR amplified from the genomic DNA of EFE7942 strain using primers NS15 and NS16, and inserted into the neutral site 1 of the genome of Synechococcus GD7942, resulting in strain GD-EFE7942. The complete segregation of genomes was verified via colony PCR, followed by DNA sequencing of the PCR products amplified using primers (listed in Table S3) flanking the modified regions of the cyanobacterial genomes.

SDS-PAGE and Western blotting. A protocol from a previous study was used. Briefly, when the $\mathrm{OD}_{730}$ of cyanobacterial culture reached $0.5-1.0$, approximately $5 \mathrm{OD}_{730} \cdot \mathrm{mL}$ (i.e., $10 \mathrm{~mL}$ if the $\mathrm{OD}_{730}$ of the culture equals 0.5 ) of cells were collected via centrifugation at $3220 \times \mathrm{g}, 24^{\circ} \mathrm{C}$ for $5 \mathrm{~min}$ and removal of supernatants. The cell pellets were stored at $-80^{\circ} \mathrm{C}$ until use. Upon running SDS-PAGE, cells were resuspended with $0.5 \mathrm{~mL}$ of cold $0.1 \mathrm{M}$ potassium phosphate buffer $(\mathrm{pH} 7.0)$ supplemented with DTT $(0.2$ $\mathrm{mM}$ ) and Halt Protein Inhibitor Cocktail (Thermo Fisher Scientific, MA, USA), and mixed with $0.2 \mathrm{~g} \mathrm{0.1-mm-}$ diameter acid-washed glass beads, and then subjected to bead-beating at $4^{\circ} \mathrm{C}$ for 5 minutes using the Digital Disruptor Genie (Scientific Industries, Inc., NY, USA). The cell lysate was centrifuged at $4{ }^{\circ} \mathrm{C}, 18000 \times \mathrm{g}$ for 10 min, and then the supernatant containing soluble proteins was transferred into a new Eppendorf tube placed on ice. The protein concentrations were estimated using the Bradford assay (Thermo Fisher Scientific, MA, USA). Then, $2.5 \mu$ g protein from each sample was mixed with 2x SDS-PAGE sample buffer ( $950 \mu$ BioRad 2x Laemmli Sample Buffer $+50 \mu \mathrm{BME}$ ) in a PCR tube and incubated at $99^{\circ} \mathrm{C}$ for $5 \mathrm{~min}$ using a thermocycler. Samples were then loaded onto Mini-PROTEAN® TGX Stain-Free ${ }^{\text {TM }}$ precast gels (Bio-Rad Laboratories, CA, USA), and electrophoresis was conducted at $150 \mathrm{~V}$ for about $45 \mathrm{~min}$. Gels were imaged using UV excitation in a FluorChem Q imager (ProteinSimple, CA, USA).

Western blotting was conducted using Pierce ${ }^{\mathrm{TM}}$ G2 Fast Blotter (Thermo Fisher Scientific, MA, USA). HisProbe $^{\text {TM}}$-HRP Conjugate (Thermo Fisher Scientific, MA, USA) was used as the antibody (at 1:500 dilution) 
to detect the SII1077-His. The chemiluminescent blots were imaged using FluorChem Q imager (ProteinSimple, CA, USA).

In vitro enzyme activity assay. His-tagged SII1077 i.e., SII1077-His, was first purified from Synechocystis PB816W. PB816W was grown in $250 \mathrm{~mL} \mathrm{mBG11}$ medium under $50 \mu \mathrm{E} \mathrm{m}^{-2} \mathrm{~s}^{-1}$ until an $\mathrm{OD}_{730}$ of about 3 , and then cells were harvested via centrifugation at $3220 \times \mathrm{g}, 24^{\circ} \mathrm{C}$ for $10 \mathrm{~min}$ followed by removal of supernatants. The cell pellets were stored at $-80^{\circ} \mathrm{C}$. Cells were subsequently resuspended with $10 \mathrm{~mL}$ of cold $0.1 \mathrm{M}$ potassium phosphate buffer $(\mathrm{pH} 7.0)$ supplemented with DTT $(0.2 \mathrm{mM})$ and Halt Protein Inhibitor Cocktail (Thermo Fisher Scientific, MA, USA), and lysed by sonication in an ice-water bath using a Q500 Sonicator (Qsonica L.L.C, CT, USA) programed for 100 cycles of 3-sec-on-3-sec-off at an amplitude of $20 \%$. The cell lysate was centrifuged at $4^{\circ} \mathrm{C}, 8000 \times \mathrm{g}$ for $10 \mathrm{~min}$, and then the supernatant containing soluble proteins was run through His GraviTrap (GE Healthcare) to purify SIl1077-His following the user manual. Briefly, the purification column containing 1-mL Ni sepharose was first equilibrated with $10 \mathrm{~mL}$ binding buffer ( $20 \mathrm{mM}$ sodium phosphate, $500 \mathrm{mM} \mathrm{NaCl}, 45 \mathrm{mM}$ imidazole, $\mathrm{pH} 7.4$ ), and then was loaded with the approximately $10 \mathrm{~mL}$ cleared cell lysate. After all of the lysate went through the $\mathrm{Ni}$ sepharose, the sepharose was washed twice, with $10 \mathrm{~mL}$ and $5 \mathrm{~mL}$ of the binding buffer, respectively. Ultimately, $3 \mathrm{~mL}$ elution buffer (20 mM sodium phosphate, $500 \mathrm{mM} \mathrm{NaCl}, 500 \mathrm{mM}$ imidazole, $\mathrm{pH}$ 7.4) was applied to the purification column to elute SII1077-His.

$0.4 \mathrm{~mL}$ purified Sll1077-His ( $\left.3.5 \mathrm{mg} \mathrm{mL}^{-1}\right)$ was mixed with $30 \mu \mathrm{L}$ guanidine $(1 \mathrm{M})$ dissolved in $5.6 \mathrm{~mL}$

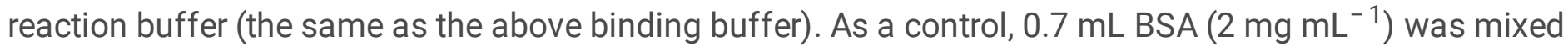
with $30 \mu \mathrm{L}$ guanidine $(1 \mathrm{M})$ dissolved in $5.3 \mathrm{~mL}$ reaction buffer. The reaction mixtures were incubated on a rotary shaker at $30{ }^{\circ} \mathrm{C}$ for 12 hours. Subsequently, the samples were passed through $30-k D$ membrane via centrifugation at $5000 \times \mathrm{g}, 24^{\circ} \mathrm{C}$, and $1.5 \mathrm{~mL}$ flow-through was freeze-dried under cryogenic vacuum. To detect urea in the samples, the dried samples were derivatized via reacting with $70 \mu \mathrm{L}$ of MTBSTFA $+1 \%$ TBDMCS (Regis Technologies, Inc.) at $70{ }^{\circ} \mathrm{C}$ for $30 \mathrm{~min}$. The derivatized samples were centrifuge at $17000 \mathrm{x}$ $\mathrm{g}$, room temperature for $5 \mathrm{~min}$, and then $1 \mu \mathrm{L}$ the supernatants were analyzed on GC-MS using a method adapted from a previous study 45 .

In vitro substrate preference assay for Sll1077. Synechococcus 7942 and GD7942 strains were grown in 250-mL flasks each containing $60 \mathrm{~mL} \mathrm{mBG11}$ medium supplemented with $50 \mathrm{mM} \mathrm{NaHCO}_{3}$ on a rotary shaker at $130 \mathrm{rpm}$, under $1 \% \mathrm{CO} 2,60 \mu \mathrm{E} \mathrm{m}^{-2} \mathrm{~s}^{-1}$ until $\mathrm{OD}_{730}$ reached about 1.5. Then $60 \mathrm{OD}_{730} \cdot \mathrm{mL}$ of cells were harvested, and centrifuged at $4700 \times \mathrm{g}, 24{ }^{\circ} \mathrm{C}$ for $10 \mathrm{~min}$. The supernatants were discarded and the cell pellets were kept at $-80^{\circ} \mathrm{C}$ until use. Subsequently, cell pellets were resuspended with $1 \mathrm{~mL} 100 \mathrm{mM}$ Tris• $\mathrm{HCl}$ (pH8.0) containing $1 \mathrm{mM}$ DTT and $1 \times$ Halt Protein Inhibitor Cocktail (Thermo Fisher Scientific, MA, USA), and lysed by sonication in an ice-water bath. The lysates were then centrifuged at $4{ }^{\circ} \mathrm{C}, 17000 \times \mathrm{g}$ for 30 $\mathrm{min}$. Then the cell extract (supernatants) were used for the following in vitro assay: $1.54 \mathrm{~mL} 100 \mathrm{mM}$

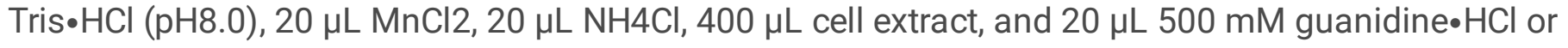
agmatine $\cdot \mathrm{HCl}$ (with a total reaction volume of $2 \mathrm{~mL}$ ). All the components but the cell extract in each reaction mix were mixed together and incubated in a $30{ }^{\circ} \mathrm{C}$ water bath for about 15 min before the cell extract was added into the reaction mix to start the assay. In the control experiments, cell extract were replaced by the 
$400 \mu \mathrm{L} 100 \mathrm{mM}$ Tris $\bullet \mathrm{HCl}(\mathrm{pH} 8.0)$ containing $1 \mathrm{mM} \mathrm{DTT}$ and 1 x Halt Protein Inhibitor Cocktail. $0.5 \mathrm{~mL}$ sample was taken from the reaction mixes at 0,2 and $12 \mathrm{~h}$ time points, and were immediately mixed with 50 $\mu \mathrm{L} 2 \mathrm{~N} \mathrm{HCl}$ to quench any enzymatic activity. $50 \mu \mathrm{L} 2 \mathrm{~N} \mathrm{NaOH}$ was then added the samples to neutralize the $\mathrm{pH}$ followed by storage at $-20^{\circ} \mathrm{C}$. After all samples were collected, $150 \mu \mathrm{L}$ of each sample was used for quantification of urea using GC-MS and another aliquot of $150 \mu \mathrm{L}$ was used for quantification of guanidine and agmatine using HPLC. For GC-MS quantification of urea, each sample was mixed with $600 \mu \mathrm{L}$ methanol, vortexed, added $150 \mu \mathrm{L}$ chloroform, vortexed, $450 \mu \mathrm{L}$ water, vortexed, and then centrifuged at $17000 \times \mathrm{g}$ for 2 min. The aqueous layer $(\sim 1.2 \mathrm{~mL})$ were transferred into a clean Eppendorf tube, air-dried over night and then lyophilized before being derivatized with MTBSTFA + 1\% TBDMCS (Regis Technologies, Inc.) at $70{ }^{\circ} \mathrm{C}$ for 30 min, and subsequently run on GC-MS for analysis of urea. A series of concentrations of urea standards were dissolved in the in vitro enzyme assay buffer, lyophilized and derivatized side by side with the enzyme assay samples in order to establish a calibration curve to quantify the urea. For HPLC quantification of guanidine and agmatine, samples were subjected to methanol/chloroform extraction and air-dried, and then resuspended with $750 \mu \mathrm{L}$ of water before being loaded on to HPLC using a method described below.

Quantification of guanidine and agmatine using HPLC. Guanidine was quantified using a protocol modified from a previous method ${ }^{26}$. Briefly, guanidine hydrochloride and agmatine standard solutions and biological samples were passed through $0.2 \mu \mathrm{m}$ diameter membrane filters and then were analyzed using an Agilent 1200 Series HPLC (Agilent, USA) equipped with a Multi-Wavelength Detector and a set of Dionex lonPac ${ }^{\mathrm{TM}}$ CS14 cation-exchange guard ( $4 \mathrm{~mm}$ x $50 \mathrm{~mm}$ ) and analytical columns ( $4 \mathrm{~mm} \times 250 \mathrm{~mm}$; Thermo Fisher Scientific, MA, USA). The column temperature was held at $30^{\circ} \mathrm{C}$. The mobile phase was $20 \mathrm{mM}$ methanesulfonic acid dissolved in $5 \%$ acetonitrile in water, and it was pumped through the column at a constant flow rate of $1.0 \mathrm{~mL} \mathrm{~min}^{-1}$ for $30 \mathrm{~min}$. Sample injection volume was $50 \mu \mathrm{L}$. Guanidine and agmatine were eluted at around $3.7 \mathrm{~min}$ and $7.9 \mathrm{~min}$, respectively, and were monitored by their absorbance at $195 \mathrm{~nm}$.

Guanidine tolerance and degradation test. For guanidine tolerance test, cyanobacterial strains were grown in $20 \mathrm{~mL}$ mBG11 medium supplemented with 0-1 mM guanidine and $50 \mathrm{mM} \mathrm{NaHCO}_{3}$. For guanidine degradation test, cyanobacterial strains were grown in $10 \mathrm{~mL}$ mBG11 free of nitrate while supplemented with $50 \mathrm{mM} \mathrm{NaHCO}_{3}$ and $5 \mathrm{mM}$ or $1 \mathrm{mM}$ guanidine chloride, under constant light of $50 \mu \mathrm{E} \mathrm{m} \mathrm{m}^{-2} \mathrm{~s}^{-1}$ on a rotary shaker at $150 \mathrm{rpm}$ and $30^{\circ} \mathrm{C}$. Every day, $1 \mathrm{~mL}$ of culture was sampled for reading $\mathrm{OD}_{730}$ and then transferred into an Eppendorf tube and centrifuged at $17000 \mathrm{xg}$ at room temperature for $2 \mathrm{~min}$. The supernatants were stored at $-20^{\circ} \mathrm{C}$ for later analysis of guanidine.

Production of ethylene from engineered Synechococcus strains. The Synechococcus EFE7942, GD-EFE7942 and WT (a negative control) strains were grown in mBG11 supplemented with $10 \mathrm{mM} H E P E S-N a O H ~(p H 8.2)$ and $20 \mathrm{mM} \mathrm{NaHCO}_{3}$ at $35^{\circ} \mathrm{C}$ until $\mathrm{OD}_{730}$ reached approximately 1.0. Subsequently, each strain was inoculated into $50 \mathrm{~mL}$ fresh medium with an initial $\mathrm{OD}_{730}$ of about 0.05 , and grown under continuous light of $100 \mu \mathrm{E} \mathrm{m}^{-2} \mathrm{~s}^{-1}$ at $30{ }^{\circ} \mathrm{C}$ aerated with $1 \% \mathrm{CO}_{2}$ at a rate of $50 \mathrm{~mL} \mathrm{~min}^{-1}$. Every day, $2 \mathrm{~mL}$ culture was sampled for ethylene productivity assay, measurement of $O D_{730}$ and guanidine analysis. After every three 
days of cultivation, appropriate volumes of cultures were centrifuged and resuspended with $50 \mathrm{~mL}$ fresh medium to an initial $\mathrm{OD}_{730}$ of about 0.05 .

Measurement of ethylene produced from cyanobacteria. $1 \mathrm{~mL}$ cyanobacterial culture was transferred into a $17-\mathrm{mL}$ glass test tube, sealed immediately with rubber stopper, and incubated under $100 \mu \mathrm{E} \mathrm{m}-2 \mathrm{~s}^{-1}$ at $30{ }^{\circ} \mathrm{C}$ with shaking. After $3 \mathrm{~h}$ incubation, $250 \mu \mathrm{L}$ gas was sampled from the headspace of the test tube using a sample-lock syringe and injected into the Shimadzu GC-2010 system equipped with a flame ionization detector (FID) and a RESTEK column (length, $30.0 \mathrm{~m}$; inner diameter, $0.32 \mathrm{~mm}$; film thickness, $5 \mu \mathrm{m}$ ). The GCFID was operated under the following conditions: carrier gas, helium; inlet temperature, $200{ }^{\circ} \mathrm{C}$; split ratio, 25 ; inlet total flow, $40.4 \mathrm{~mL} / \mathrm{min}$; Pressure, $79 \mathrm{kPa}$; column flow, $1.53 \mathrm{~mL} / \mathrm{min}$; linear velocity, $32.1 \mathrm{~cm} / \mathrm{sec}$ (Flow Control Mode); purge flow, $0.5 \mathrm{~mL} / \mathrm{min}$; column temperature, $130^{\circ} \mathrm{C}$; equilibration time, 2 min; hold time, 2 min; FID temperature, $200^{\circ} \mathrm{C}$; sampling rate, $40 \mathrm{msec}$; stop time, $2 \mathrm{~min}$; FID makeup gas, He; FID makeup flow, $30 \mathrm{~mL} / \mathrm{min}$; $\mathrm{H} 2$ flow, $40 \mathrm{~mL} / \mathrm{min}$; air flow: $400 \mathrm{~mL} / \mathrm{min}$.

Shotgun proteomics. Synechocystis 6803 and the ethylene-producing JU547 were inoculated into $3 \times 50 \mathrm{~mL}$ $\mathrm{mBG} 11$ with an initial $O D_{730}$ of 0.1 . When $\mathrm{OD}_{730}$ reached about $0.5,60 \mathrm{OD}_{730} \cdot \mathrm{mL}$ cells were collected via centrifugation at $3220 \times \mathrm{g}, 4^{\circ} \mathrm{C}$ for $5 \mathrm{~min}$. The cell pellets were washed with $25 \mathrm{~mL}$ cold wash buffer ( $50 \mathrm{mM}$ Tris $\bullet \mathrm{HCl}$, pH8.0 and $10 \mathrm{mM} \mathrm{CaCl}_{2}$ ) and centrifuged again, followed by washing with $20 \mathrm{~mL}$ and $1 \mathrm{~mL}$ washing buffer. The supernatants were discarded and cells were frozen at $-80^{\circ} \mathrm{C}$. Three biological replicates were included for each strain. Comparative proteomic analyses of Synechocystis 6803 and JU547 was conducted following our previously published method ${ }^{46}$. The sample preparation and amount of peptide loaded to the capillary column varied from that in the previous method. Briefly, cell pellets taken out of $-80^{\circ} \mathrm{C}$ were lysed by sonication with a program of 12 cycles of 10 seconds-on-2-minutes-off on ice. The supernatants were collected via centrifugation and the protein concentrations were analyzed using Bradford assay (Thermo Scientific, Rockford, IL). Then, $75 \mu \mathrm{g}$ of total protein for each sample was used for downstream proteomic sample preparation following the same procedure as described before.

\section{Declarations}

\section{Acknowledgements}

This work was authored in part by Alliance for Sustainable Energy, LLC, the manager and operator of the National Renewable Energy Laboratory for the U.S. Department of Energy (DOE) under Contract No. DEAC36-08G028308. Funding provided by DOE Office of Energy Efficiency and Renewable Energy BioEnergy Technologies Office (B.W., J.Yu.). This study was supported in part by the DOE Genomic Science Program under award DE-SC0019404 (B.W., Y.X., C.H.J., J.D.Y.), DE-SC0019388 (B.W., J.D.Y), and DE-SC0018344 (B.W., J.D.Y), and grants from the NIH/NIGMS (R37 GM067152 and R01 GM107434 to C.H.J.), and by DEAR0000203 (X.W., J.Yuan.). The views expressed in the article do not necessarily represent the views of the DOE or the U.S. Government. The U.S. Government retains and the publisher, by accepting the article for publication, acknowledges that the U.S. Government retains a nonexclusive, paid-up, irrevocable, worldwide license to publish or reproduce the published form of this work, or allow others to do so, for U.S. Government purposes. 


\section{Competing interests}

A provisional patent application has been filed based upon this work.

\section{Authors' Contributions}

B.W. and J.Yu. conceived the work. B.W. designed and performed most of the experiments and drafted the manuscript. Y.X. constructed recombinant Synechococcus elongatus strains. X.W. and J.Yuan performed proteomic analysis. C.H.J and J.D.Y. performed discussion, critical review and revision of the manuscript. All authors read and approved the manuscript.

\section{References}

1. Güthner, T., Mertschenk, B. \& Schulz, B. in Ullmann's Encyclopedia of Industrial Chemistry (ed (Ed.)) (Wiley-VCH Verlag GmbH \& Co. KGaA, 2006).

2. Zhang, Z., Nyborg, M., Worsley, M., Worsley, K. M. \& Gower, D. A. Guanidine sulphate: Slow release of mineral nitrogen during incubation in soil. Commun. Soil Sci. Plant Anal. 23, 431-439, doi:10.1080/00103629209368601 (1992).

3. Putnam, D. F. Composition and concentrative properties of human urine. Report No. NASA-CR-1802, DAC-61125-F, (1971).

4. Natelson, S. \& Sherwin, J. E. Proposed mechanism for urea nitrogen re-utilization: relationship between urea and proposed guanidine cycles. Clin. Chem. 25, 1343-1344 (1979).

5. Nelson, J. W., Atilho, R. M., Sherlock, M. E., Stockbridge, R. B. \& Breaker, R. R. Metabolism of Free Guanidine in Bacteria Is Regulated by a Widespread Riboswitch Class. Mol. Cell 65, 220-230, doi:10.1016/j.molcel.2016.11.019 (2017).

6. Lewis, C. A., Jr. \& Wolfenden, R. The nonenzymatic decomposition of guanidines and amidines. J. Am. Chem. Soc. 136, 130-136, doi:10.1021/ja411927k (2014).

7. Mitchell, W. R. Biodegradation of guanidinium ion in aerobic soil samples. Bull Environ Contam Toxicol 39, 974-981 (1987).

8. Sherlock, M. E., Malkowski, S. N. \& Breaker, R. R. Biochemical Validation of a Second Guanidine Riboswitch Class in Bacteria. Biochemistry 56, 352-358, doi:10.1021/acs.biochem.6b01270 (2017).

9. Sherlock, M. E. \& Breaker, R. R. Biochemical Validation of a Third Guanidine Riboswitch Class in Bacteria. Biochemistry 56, 359-363, doi:10.1021/acs.biochem.6b01271 (2017).

10. Lenkeit, F., Eckert, I., Hartig, J. S. \& Weinberg, Z. Discovery and characterization of a fourth class of guanidine riboswitches. Nucleic Acids Res. 48, 12889-12899, doi:10.1093/nar/gkaa1102 (2020).

11. Salvail, H., Balaji, A., Yu, D., Roth, A. \& Breaker, R. R. Biochemical Validation of a Fourth Guanidine Riboswitch Class in Bacteria. Biochemistry 59, 4654-4662, doi:10.1021/acs.biochem.0c00793 (2020).

12. Kermani, A. A., Macdonald, C. B., Gundepudi, R. \& Stockbridge, R. B. Guanidinium export is the primal function of SMR family transporters. Proc. Natl. Acad. Sci. U. S. A. 115, 3060-3065, doi:10.1073/pnas.1719187115 (2018). 
13. Schneider, N. O. et al. Solving the conundrum: Widespread proteins annotated for urea metabolism in bacteria are carboxyguanidine deiminases mediating nitrogen assimilation from guanidine. Biochemistry, doi:10.1021/acs.biochem.0c00537 (2020).

14. Kaneko, T. et al. Sequence analysis of the genome of the unicellular cyanobacterium Synechocystis sp. strain PCC6803. II. Sequence determination of the entire genome and assignment of potential proteincoding regions. DNA Res. 3, 109-136 (1996).

15. Sekowska, A., Danchin, A. \& Risler, J. L. Phylogeny of related functions: the case of polyamine biosynthetic enzymes. Microbiology (Reading) 146 (Pt 8), 1815-1828, doi:10.1099/00221287-146-81815 (2000).

16. Cunin, R., Glansdorff, N., Pierard, A. \& Stalon, V. Biosynthesis and metabolism of arginine in bacteria. Microbiol. Rev. 50, 314-352 (1986).

17. Eckert, C. et al. Ethylene-forming enzyme and bioethylene production. Biotechnol Biofuels 7, 33, doi:10.1186/1754-6834-7-33 (2014).

18. Guerrero, F., Carbonell, V., Cossu, M., Correddu, D. \& Jones, P. R. Ethylene synthesis and regulated expression of recombinant protein in Synechocystis sp. PCC 6803. PLoS One 7, e50470, doi:10.1371/journal.pone.0050470 (2012).

19. Ungerer, J. et al. Sustained photosynthetic conversion of $\mathrm{CO} 2$ to ethylene in recombinant cyanobacterium Synechocystis 6803. Energy Environ. Sci. 5, 8998-9006, doi:10.1039/c2ee22555g (2012).

20. Zhu, T., Xie, X., Li, Z., Tan, X. \& Lu, X. Enhancing photosynthetic production of ethylene in genetically engineered Synechocystis sp. PCC 6803. Green Chem. 17, 421-434, doi:10.1039/C4GC01730G (2015).

21. Xiong, W. et al. The plasticity of cyanobacterial metabolism supports direct $\mathrm{CO} 2$ conversion to ethylene. Nature Plants 1, 15053, doi:10.1038/nplants.2015.53 (2015).

22. Wang, J. P. et al. Metabolic engineering for ethylene production by inserting the ethylene-forming enzyme gene (efe) at the 16S rDNA sites of Pseudomonas putida KT2440. Bioresour. Technol. 101, 6404-6409, doi:10.1016/j.biortech.2010.03.030 (2010).

23. Sakai, M., Ogawa, T., Matsuoka, M., Fukuda, H. Photosynthetic conversion of carbon dioxide to ethylene by the recombinant cyanobacterium, Synechococcus sp. PCC 7942, which harbors a gene for the ethylene-forming enzyme of Pseudomonas syringae. Journal of Fermentation and Bioengineering 84, 434-443, doi:https://doi.org/10.1016/S0922-338X(97)82004-1 (1997).

24. Takahama, K., Matsuoka, M., Nagahama, K. \& Ogawa, T. Construction and analysis of a recombinant cyanobacterium expressing a chromosomally inserted gene for an ethylene-forming enzyme at the psbAl locus. J Biosci Bioeng 95, 302-305, doi:10.1016/s1389-1723(03)80034-8 (2003).

25. Sengupta, A. et al. Photosynthetic Co-Production of Succinate and Ethylene in A Fast-Growing Cyanobacterium, Synechococcus elongatus PCC 11801. Metabolites 10, doi:10.3390/metabo10060250 (2020).

26. Wang, B. et al. Photosynthetic production of the nitrogen-rich compound guanidine. Green Chem. 21, 2928-2937, doi:10.1039/C9GC01003C (2019). 
27. Satishchandran, C. \& Boyle, S. M. Purification and properties of agmatine ureohydrolyase, a putrescine biosynthetic enzyme in Escherichia coli. J. Bacteriol. 165, 843-848, doi:10.1128/jb.165.3.843-848.1986 (1986).

28. Veaudor, T., Cassier-Chauvat, C. \& Chauvat, F. Genomics of Urea Transport and Catabolism in Cyanobacteria: Biotechnological Implications. Front. Microbiol. 10, 2052, doi:10.3389/fmicb.2019.02052 (2019).

29. Nagahama, K. et al. Purification and properties of an ethylene-forming enzyme from Pseudomonas syringae pv. phaseolicola PK2. J. Gen. Microbiol. 137, 2281-2286, doi:10.1099/00221287-137-10-2281 (1991).

30. Ishihara, K. et al. Overexpression and in vitro reconstitution of the ethylene-forming enzyme from Pseudomonas syringae. Journal of Fermentation and Bioengineering 79, 205-211, doi:https://doi.org/10.1016/0922-338X(95)90604-X (1995).

31. Wang, B., Eckert, C., Maness, P. C. \& Yu, J. A Genetic Toolbox for Modulating the Expression of Heterologous Genes in the Cyanobacterium Synechocystis sp. PCC 6803. ACS Synth Biol 7, 276-286, doi:10.1021/acssynbio.7b00297 (2018).

32. Kaneko, T. et al. Sequence analysis of the genome of the unicellular cyanobacterium Synechocystis sp. strain PCC6803. II. Sequence determination of the entire genome and assignment of potential proteincoding regions. DNA Res. 3, 109-136, doi:10.1093/dnares/3.3.109 (1996).

33. Quintero, M. J., Muro-Pastor, A. M., Herrero, A. \& Flores, E. Arginine catabolism in the cyanobacterium Synechocystis sp. Strain PCC 6803 involves the urea cycle and arginase pathway. J. Bacteriol. 182, 1008-1015, doi:10.1128/jb.182.4.1008-1015.2000 (2000).

34. Zhang, $\mathrm{H}$. et al. The cyanobacterial ornithine-ammonia cycle involves an arginine dihydrolase. Nat. Chem. Biol. 14, 575-581, doi:10.1038/s41589-018-0038-z (2018).

35. Kuchmina, E. et al. Ethylene production in Synechocystis sp. PCC 6803 promotes phototactic movement. Microbiology 163, 1937-1945, doi:10.1099/mic.0.000564 (2017).

36. Heyda, J. et al. Guanidinium can both Cause and Prevent the Hydrophobic Collapse of Biomacromolecules. Journal of the American Chemical Society 139, 863-870, doi:10.1021/jacs.6b11082 (2017).

37. Zheng, W. et al. Probing the Action of Chemical Denaturant on an Intrinsically Disordered Protein by Simulation and Experiment. Journal of the American Chemical Society 138, 11702-11713, doi:10.1021/jacs.6b05443 (2016).

38. Yang, H. J. \& Tsou, C. L. Inactivation during denaturation of ribonuclease A by guanidinium chloride is accompanied by unfolding at the active site. Biochem J 305 (Pt 2), 379-384, doi:10.1042/bj3050379 (1995).

39. Lees, H. \& Quastel, J. H. Biochemistry of nitrification in soil; nitrification of various organic nitrogen compounds. Biochem J 40, 824-828 (1946).

40. Forchhammer, K. \& Schwarz, R. Nitrogen chlorosis in unicellular cyanobacteria - a developmental program for surviving nitrogen deprivation. Environ. Microbiol. 21, 1173-1184, doi:10.1111/14622920.14447 (2019). 
41. Carbonell, V., Vuorio, E., Aro, E. M. \& Kallio, P. Enhanced stable production of ethylene in photosynthetic cyanobacterium Synechococcus elongatus PCC 7942. World J Microbiol Biotechnol 35, 77, doi:10.1007/s11274-019-2652-7 (2019).

42. Cheah, Y. E. et al. Systematic identification and elimination of flux bottlenecks in the aldehyde production pathway of Synechococcus elongatus PCC 7942. Metab. Eng. 60, 56-65, doi:10.1016/j.ymben.2020.03.007 (2020).

43. Wang, B., Yu, J., Zhang, W. \& Meldrum, D. R. Premethylation of foreign DNA improves integrative transformation efficiency in Synechocystis sp. strain PCC 6803. Appl Environ Microbio/ 81, 8500-8506, doi:10.1128/AEM.02575-15 (2015).

44. Golden, S. S., Brusslan, J. \& Haselkorn, R. Genetic engineering of the cyanobacterial chromosome. Methods Enzymol. 153, 215-231, doi:10.1016/0076-6879(87)53055-5 (1987).

45. Jazmin, L. J. et al. Isotopically nonstationary (13)C flux analysis of cyanobacterial isobutyraldehyde production. Metab. Eng. 42, 9-18, doi:10.1016/j.ymben.2017.05.001 (2017).

46. Wang, $X$. et al. Enhanced limonene production in cyanobacteria reveals photosynthesis limitations. Proc. Natl. Acad. Sci. U. S. A. 113, 14225-14230 (2016).

\section{Figures}

a

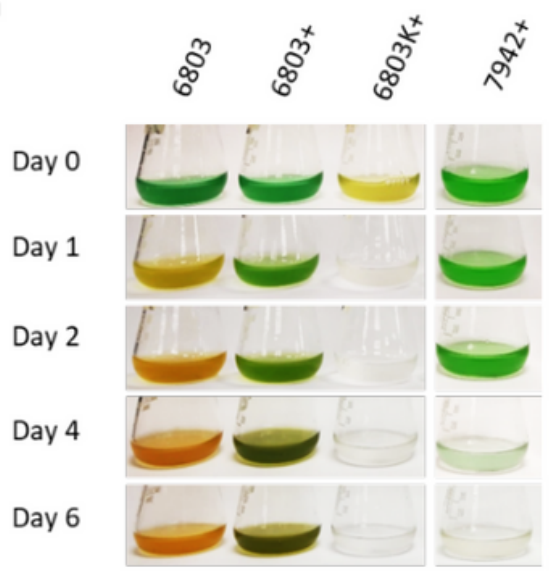

b

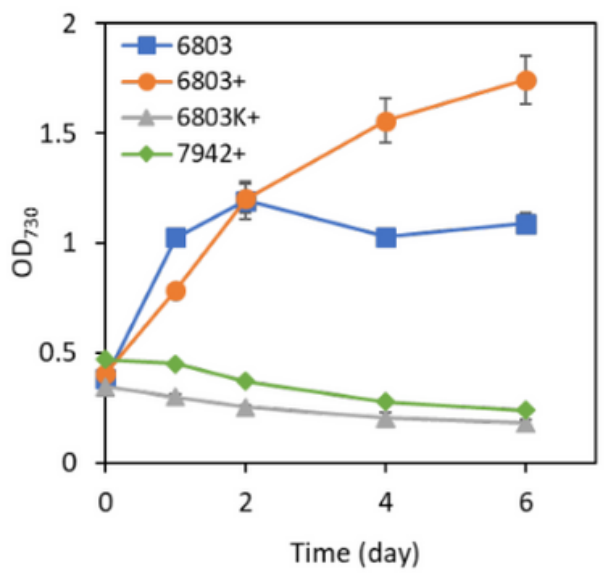

c

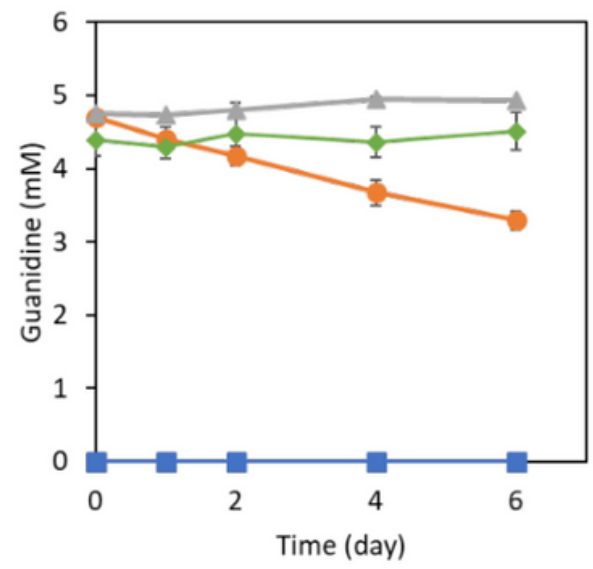

\section{Figure 1}

Varied capabilities in degrading guanidine between two model cyanobacterial species. a, Phenotypes of Synechocystis 6803 and Synechococcus 7942 grown in nitrate-deprived medium with or without guanidine. "6803", Synechocystis 6803 strain grown in nitrate-deprived medium; "6803+", Synechocystis 6803 strain grown in nitrate-deprived medium supplemented with $5 \mathrm{mM}$ guanidine. "6803K+", Synechocystis 6803 cells initially killed by heating at $95 \mathrm{oC}$ for $10 \mathrm{~min}$ and then resuspended in the nitrate-deprived medium supplemented with $5 \mathrm{mM}$ guanidine. "7942+", Synechococcus 7942 strain grown in nitrate-deprived medium supplemented with $5 \mathrm{mM}$ guanidine. $b$, Time courses of cell mass accumulation as monitored by readings 
of OD730. c, Time courses of guanidine concentrations in the culture supernatants. Data represent means and standard deviations from three biological replicates.

a

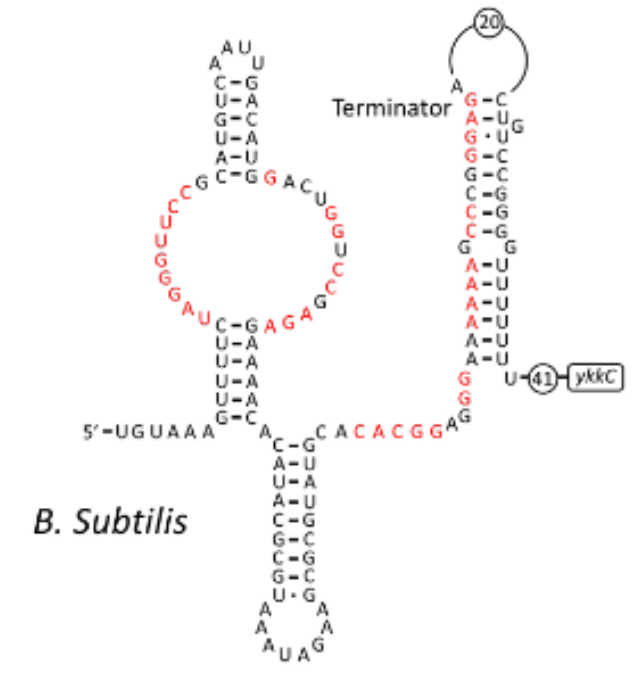

b

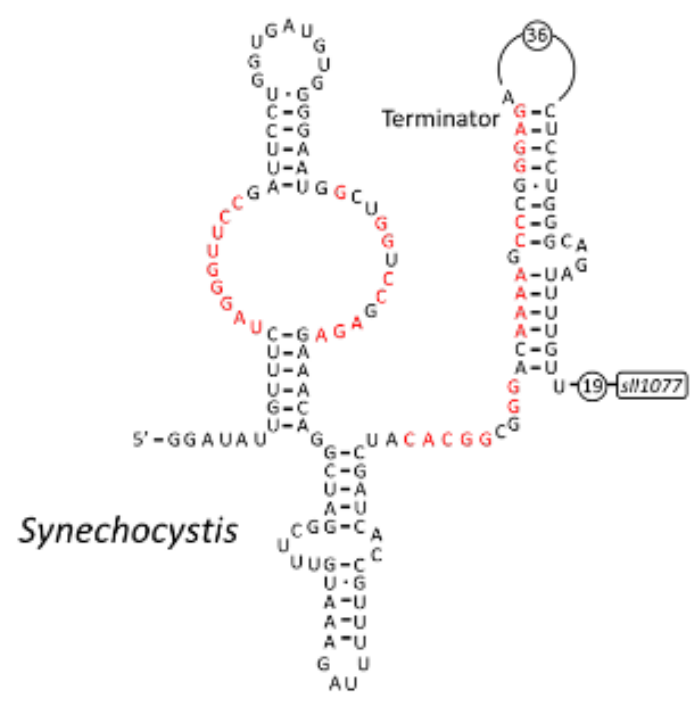

c
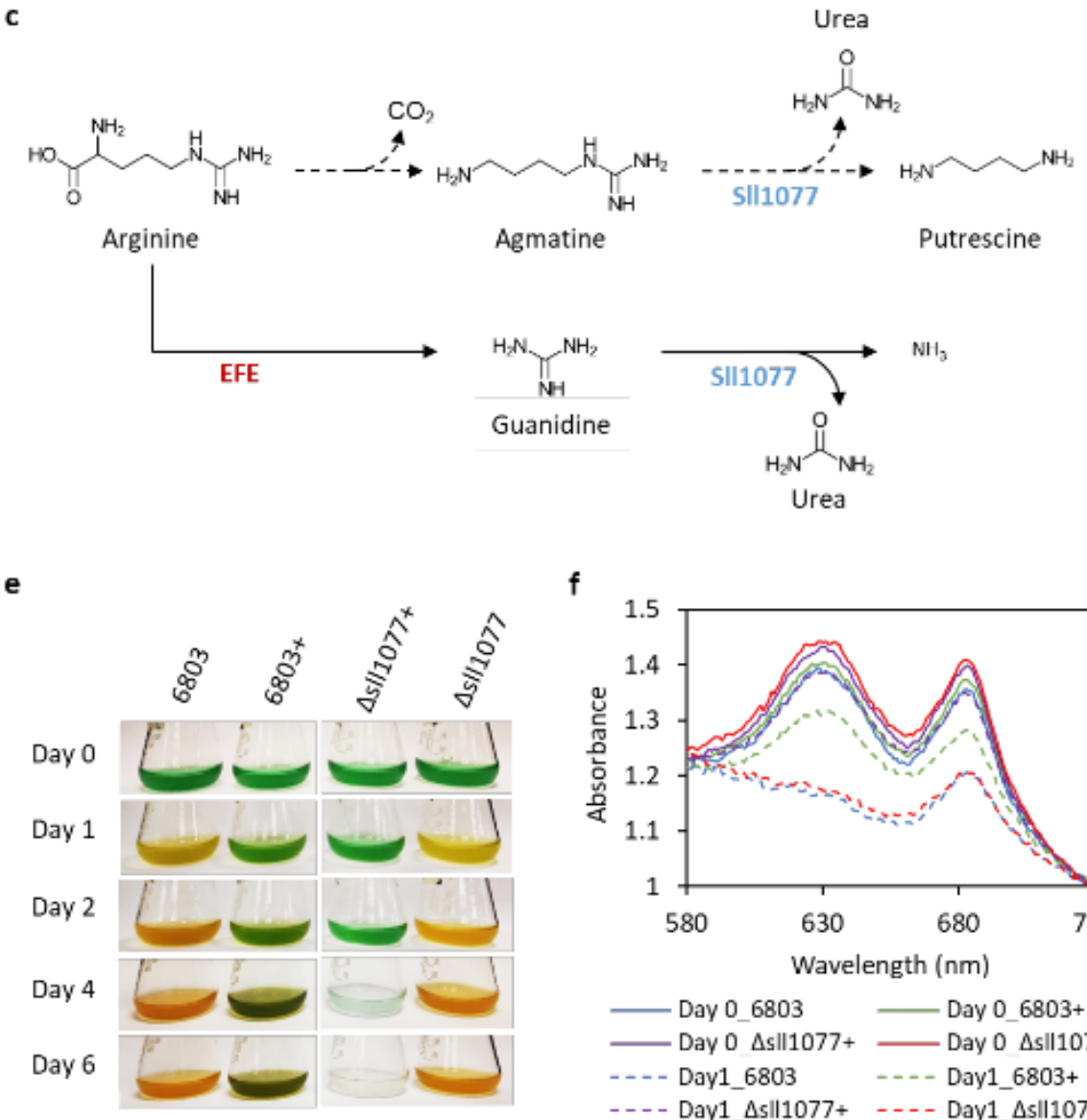

f
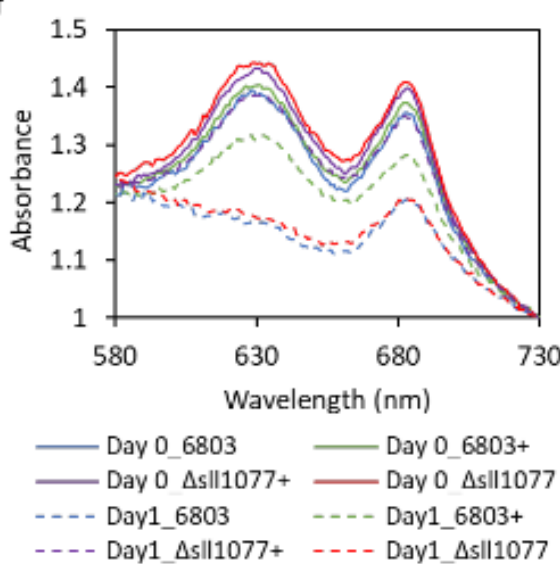

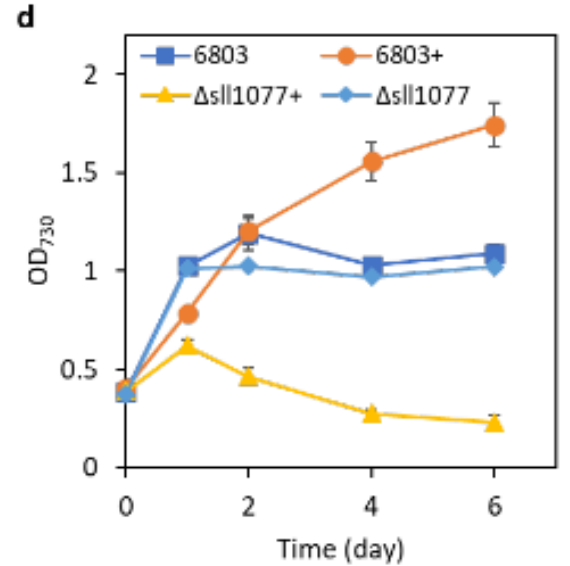

d

g

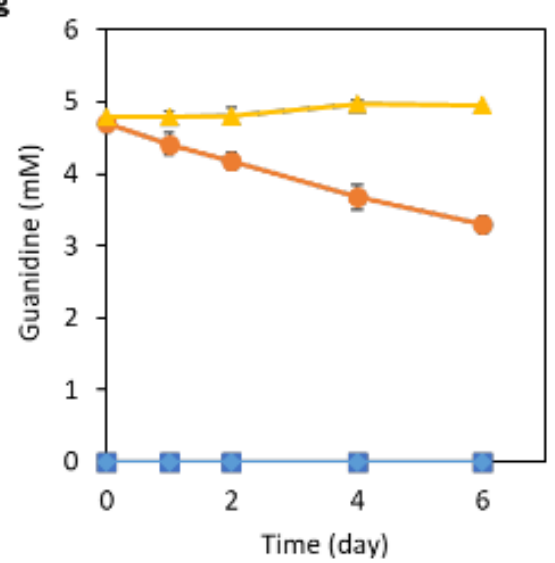

\section{Figure 2}

Gene sll1077 is responsible for guanidine degradation in Synechocystis 6803. a, The secondary RNA structure of the guanidine riboswitch upstream of the guanidine exporter encoded by the ykkC gene in Bacillus subtilis. Nucleotides in red font are $>97 \%$ conserved in type I guanidine riboswitch5. b, The secondary RNA structure of predicted guanidine riboswitch upstream of the sll1077 gene in Synechocystis 
6803. The consensus guanidine riboswitch nucleotides are depicted in red font. c, The proposed metabolic role of SIl1077 in degrading guanidine as depicted by solid arrows. Sll1077 was previously annotated as an agmatinase in one arginine degradation pathway as depicted by the dotted arrows 15 . d, Time courses of cell densities of Synechocystis 6803 and the $\Delta$ sll1077 strain (PB805W) grown in nitrate-deprived medium with or without guanidine supplementation. $5 \mathrm{mM}$ guanidine was added into the nitrate-deprived medium as indicated by " + " following the strain names. e, Phenotypes of Synechocystis 6803 and the $\Delta$ sll1077 strain (PB805W). f, Absorbance spectra of cultures at day 0 and day 1 as shown in d. Absorbance was normalized to the absorbance at $730 \mathrm{~nm}$. $\mathrm{g}$, Time courses of guanidine concentrations in the culture supernatants. Data represent means and standard derivations from three biological replicates.

\begin{tabular}{|c|c|}
\hline $5^{\prime}$ region (RBS) & 3' region \\
\hline PB806W: AATTGTGAGCGGATAACAATTTCACACAAGGAGGATATACAT & + s//1077+ CTCGAG (Xhol site) + 6xHis tag \\
\hline PB807W: AATTGTGAGCGGATAACAATTTCACACAAGGAGGAAACAT & + s//1077+ CTCGAG (Xhol site) + 6xHis tag \\
\hline PB808W: AATTGTGAGCGGATAACAATTTCACACAAGGAGGAACAGC & + s//1077+ CTCGAG (Xhol site) + 6xHis tag \\
\hline PB809W: AATTGTGAGCGGATAACAATTTCACACAAGGAGAAACAGC & + s//1077 + CTCGAG (Xhol site) + 6xHis tag \\
\hline PB810W: AATTGTGAGCGGATAACAATTTCACACAAGAAGGAGAAACAGC & + s//1077+ CTCGAG (Xhol site) + 6xHis tag \\
\hline PB811W: AATTGTGAGCGGATAACAATTTCACACAAGAAGGAGAAACATAGC & + s//1077+ CTCGAG (Xhol site) + 6xHis tag \\
\hline PB812W: AATTGTGAGCGGATAACAATTTCACACAAGGAGAAACAGC & + s//1077+ $6 \times$ His tag \\
\hline PB816W: AATTGTGAGCGGATAACAATTTCACACAAGGAGAAACAGC & + s//1077+6xHis tag + terminator \\
\hline PB817W: AATTGTGAGCGGATAACAATTTCACACAAGGAGAAACAGC & + s/l1077+ terminator \\
\hline
\end{tabular}

b

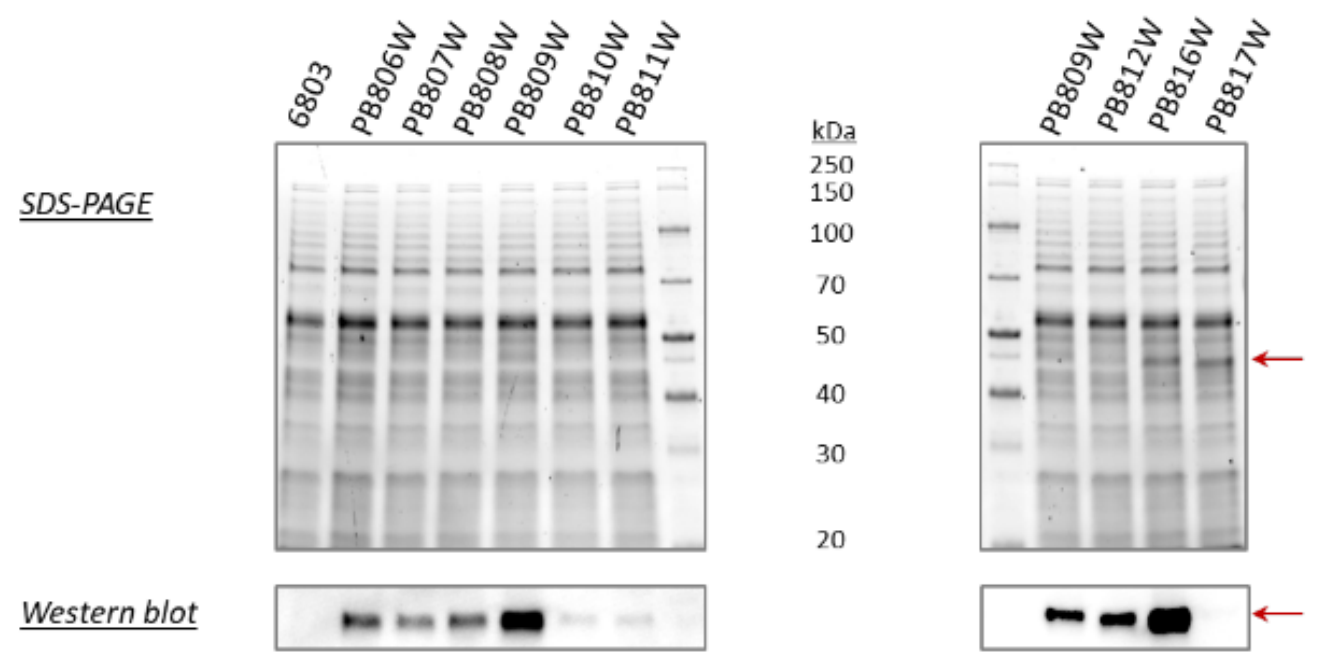

C

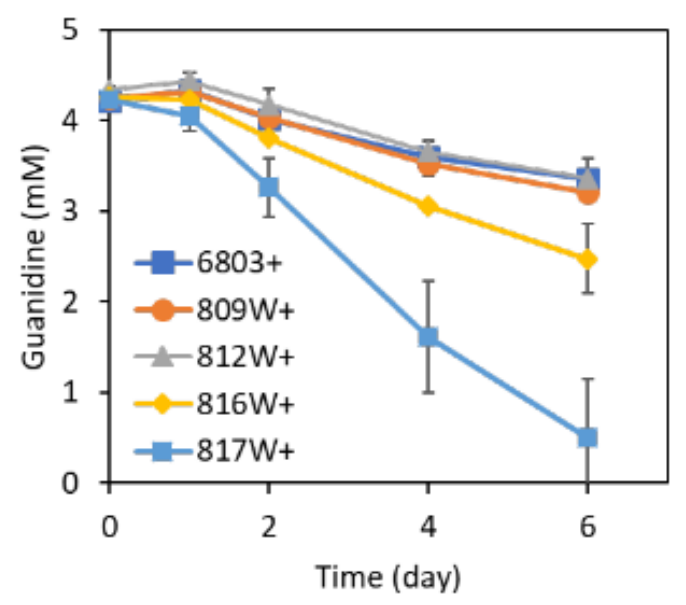

d

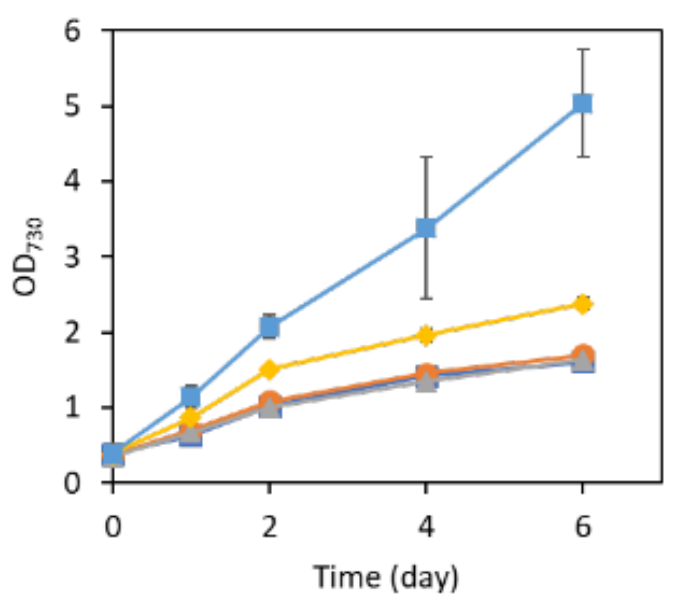

Figure 3 
Overexpression of SIl1077 accelerates guanidine degradation and promotes biomass growth in Synechocystis 6803. a, Strategies for enhancing the overexpression of gene sll1077 in Synechocystis 6803. Gene sll1077 was overexpressed driven by the tac promoter, with its RBS at the $5^{\prime}$ region and the His tag and terminator at the $3^{\prime}$ region optimized. b, SDS-PAGE and western blotting (His tag) showing the improved expression of SII1077 in Synechocystis. C, Guanidine degradation profiles of Synechocystis 6803 and sll1077-overexpressing strains. d, Cell growth curves for Synechocystis 6803 and sll1077-overexpressing strains, indicated by readings of OD730 of cell cultures. Data represent means and standard derivations from three biological replicates.

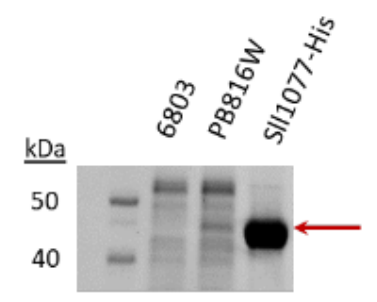

C
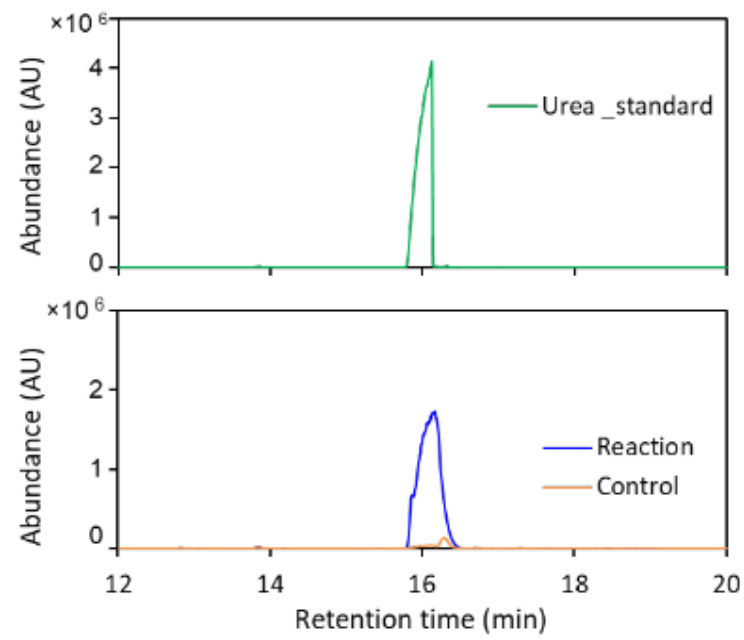

b

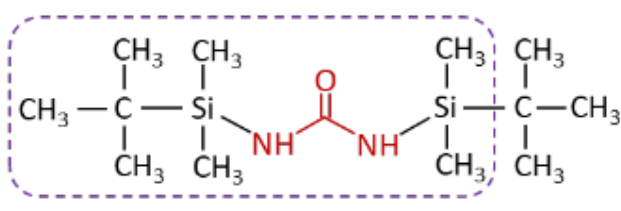

$\mathrm{m} / \mathrm{z}=231$

d

$x 10^{6}+$ El Scan (rt: 16.095 min) Urea standard.D

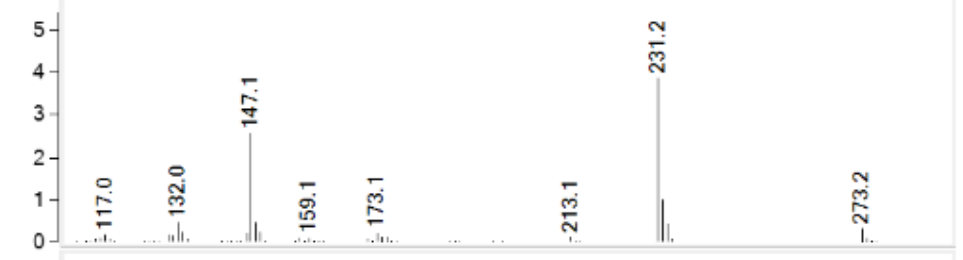

$x 10^{6}+$ El Scan (rt: $\left.16.095 \mathrm{~min}\right)$ Product from SI11077.D

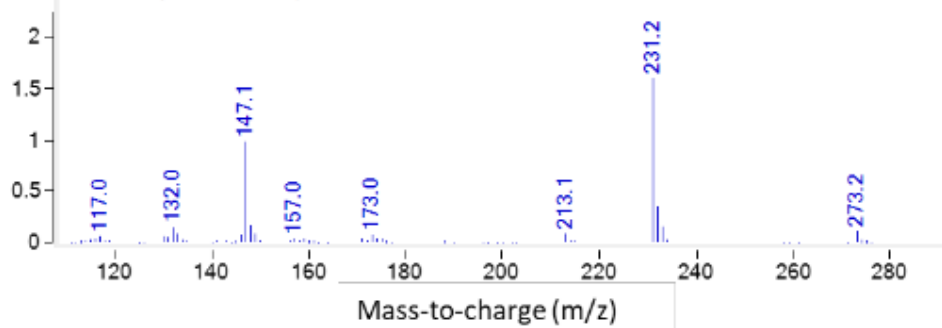

e

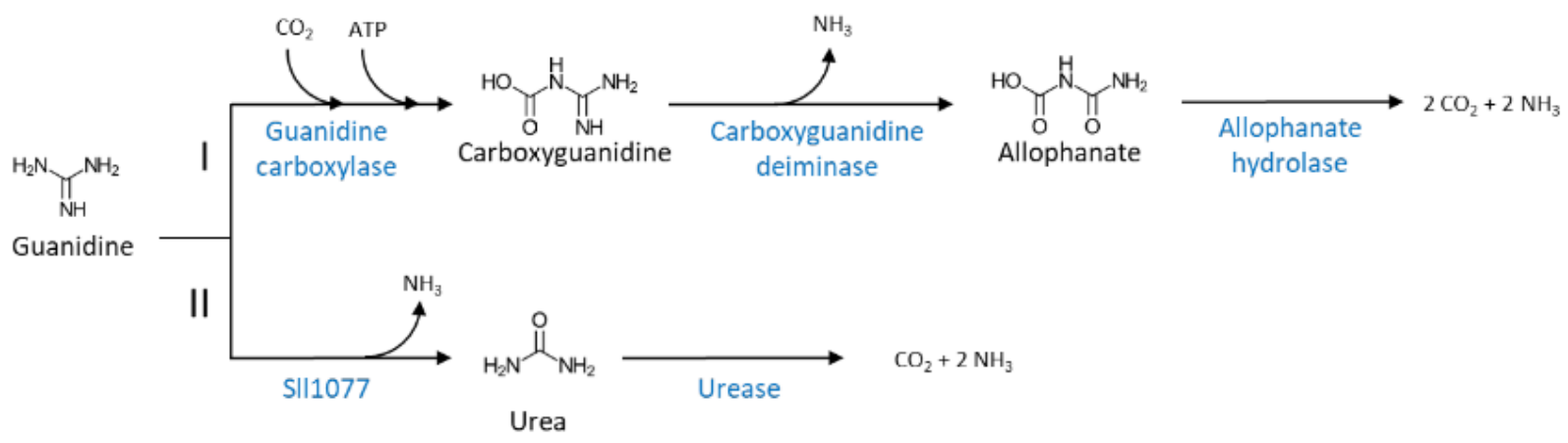

\section{Figure 4}

Confirmation of the guanidine-degrading enzyme activity of Sll1077 through an in vitro enzyme activity assay. a, SDS-PAGE showing the cell extract from Synechocystis 6803, PB816W and purified SII1077-His. b, TBDMS derivative of urea. Red font indicates the urea backbone. The boxed portion indicates the main ion detected by GC-MS. c, lon counts of ion 231 for TBDMS derivative of urea standard or the product of 
guanidine incubated with either Sll1077-His (Reaction) or bovine serum albumin (Control). d, Mass spectra of the peak at 16.095 min in c. e, Guanidine degradation pathways identified to date. Pathway I was reported previously, and pathway II is demonstrated in the current study.

a

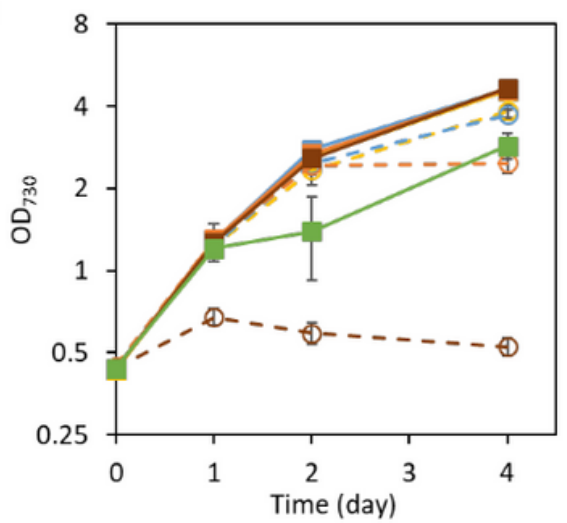

c

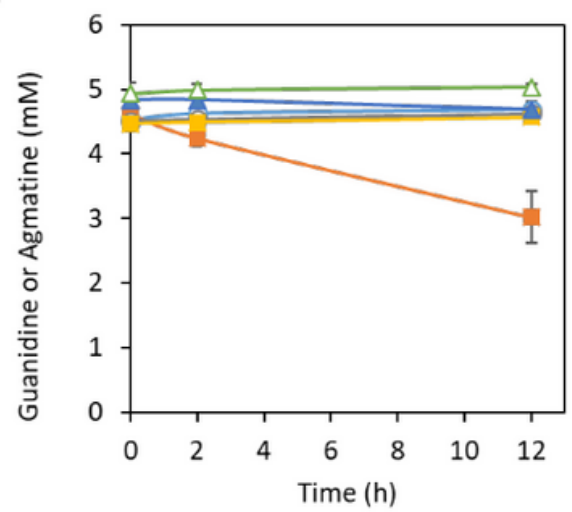

b

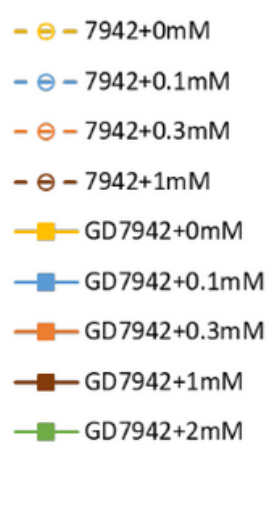

$-0-7942+5$ mM gdm

$-\mathrm{ED} 7942+5 \mathrm{mM}$ gdm

$-0-7942+5 \mathrm{mM}$ agm

- -GD7942 + 5 mM agm

$-\mathrm{Ctrl}+5 \mathrm{mM} \mathrm{gdm}$

$-\triangle \mathrm{Ctrl}+5 \mathrm{mM}$ agm

d

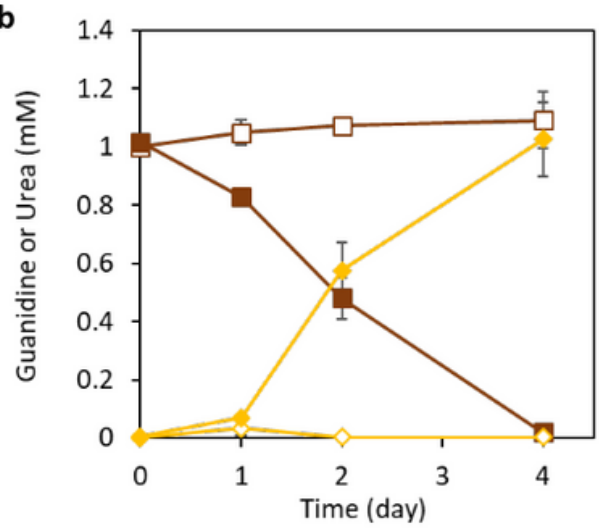

$\neg-g d m \_7942$

$\rightarrow-g d m \_G D 7942$

$\leadsto$ urea_7942

$\leadsto$ urea_GD7942

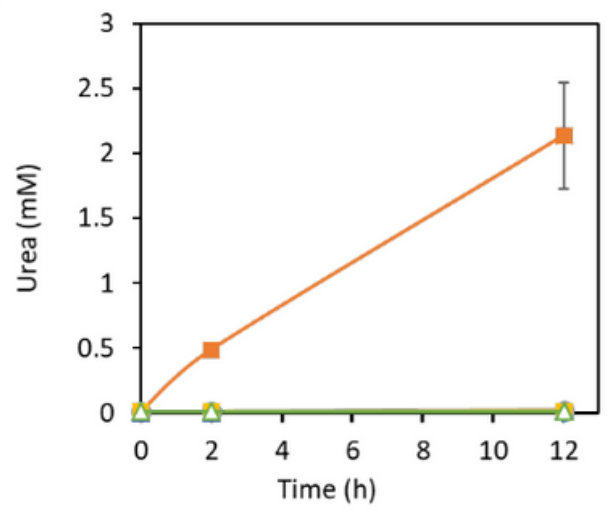

\section{Figure 5}

Expression of sll1077 improves the tolerance of Synechococcus 7942 to guanidine. a, Cell growth curves for the Synechococcus 7942 and GD7942 (+sll1077) grown with various concentrations of exogenous guanidine in the nitrate-deprived culture medium. b, Time courses of guanidine and urea concentrations in the culture supernatants of Synechococcus 7942 and GD7942 grown with $1 \mathrm{mM}$ guanidine. c, Production of urea by the cell extract of Synechococcus 7942 or GD7942 incubated with $5 \mathrm{mM}$ guanidine (gdm) or agmatine (agm). Control (Ctrl) included all the components but the cell extract. d, Concentrations of guanidine or agmatine in the enzymatic reaction mix along the time course. The symbols in panel $\mathrm{c}$ and $\mathrm{d}$ indicate the same samples. Data represent means and standard derivations from three biological replicates. 
a

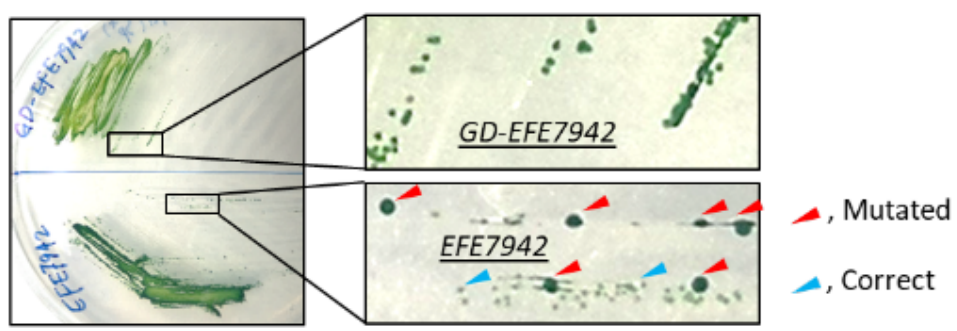

b

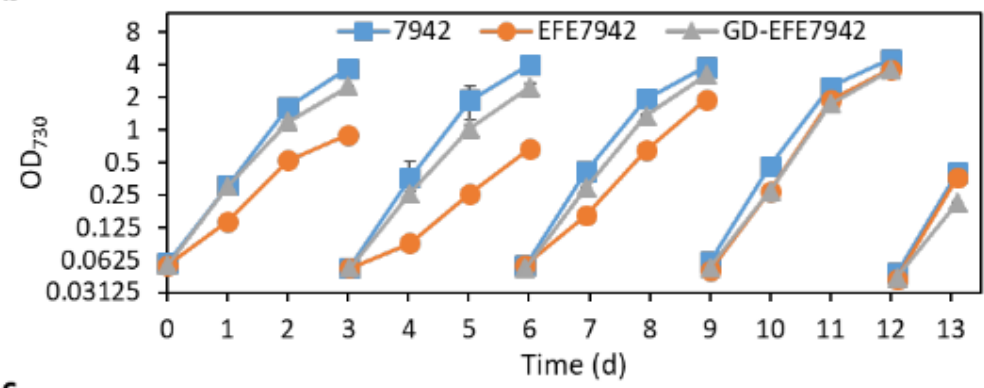

c

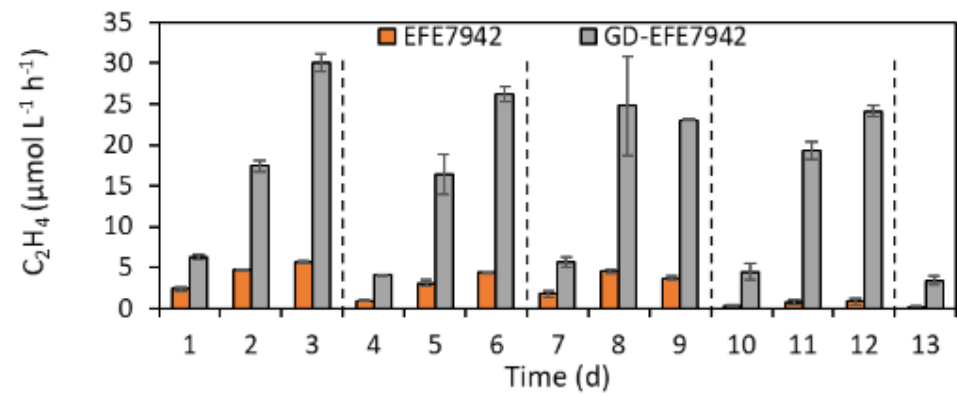

d

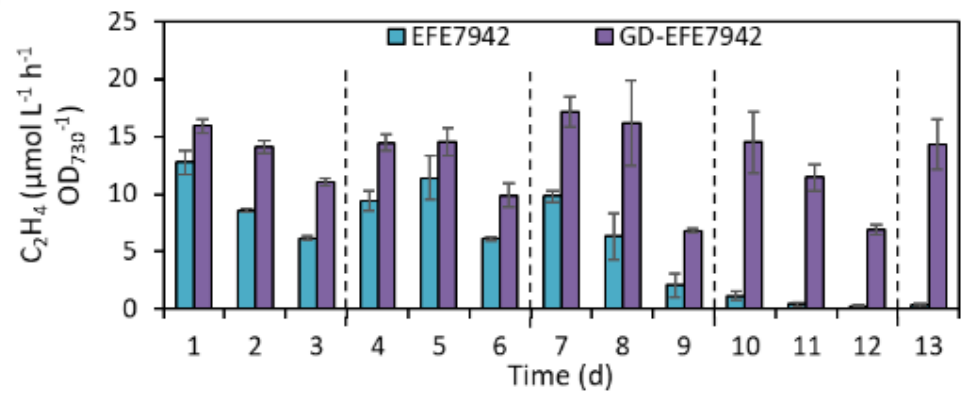

e

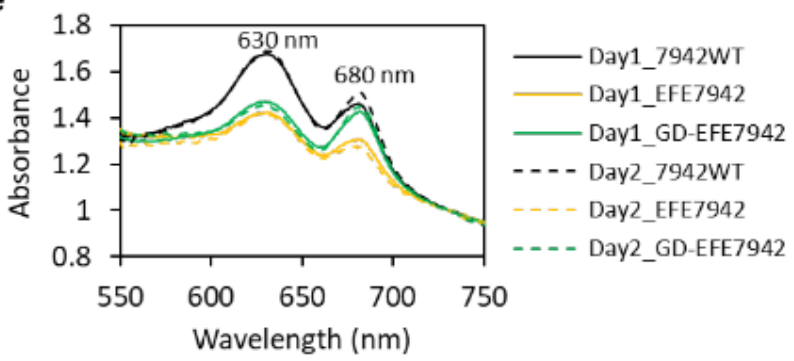

f

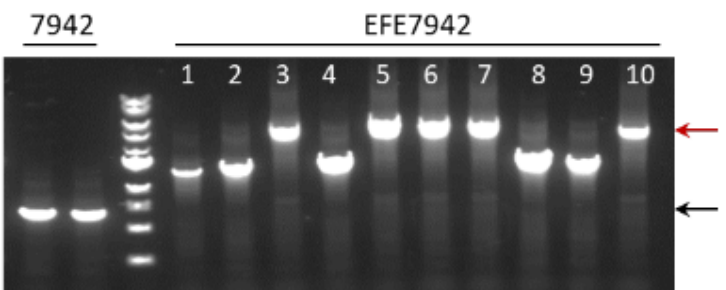

$\underline{7942}$

GD-EFE7942

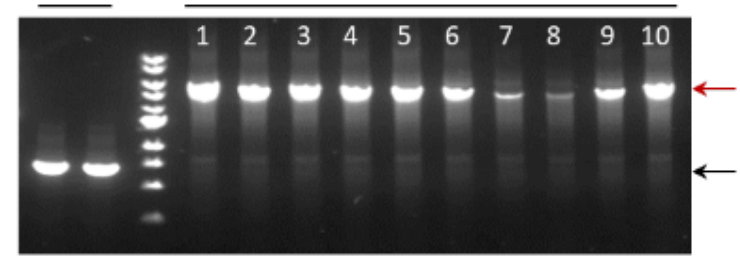

g

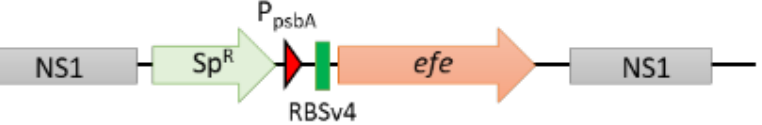

[2OG-Fe(II) oxygenase superfamily conserved region]

[2102 bp deleted in EFE7942 \#1, 2, 4, 8, 9]

[24 bp deleted in EFE7942 \#3, 5, 7, 10]

[1 bp deleted in EFE7942 \#6]

\section{Figure 6}

Expression of sll1077 in Synechococcus 7942 supports sustained high-level ethylene production. a, Colonies of strains EFE7942 and GD-EFE7942 formed on agar plates at $30 \mathrm{oC}$. DNA sequencing results revealed that for strain EFE7942, the smaller colonies indicated by cyan triangles harbored the correct EFE expression cassette, while the bigger colonies denoted by red triangles harbored mutated EFE expression cassettes; for strain GD-EFE7942, colony sizes were uniform and DNA sequencing identified no mutations around the EFE expression cassette. b, Cell growth curves in liquid cultures at $30 \mathrm{oC}$. Cultures were re-inoculated into fresh media every three days. c, Volumetric ethylene productivities of strains EFE7942 and GD-EFE7942. d, Specific ethylene productivities of strains EFE7942 and GD-EFE7942. Cultures were re-inoculated every three days. Data represent means and standard deviations from two biological replicates. e, Absorbance spectra of cultures shown in b-d at day 1 and day 2. Absorbance was normalized to the absorbance at $730 \mathrm{~nm}$. $\mathrm{f}$, Two colonies of the wild-type strain 7942, ten colonies of strain EFE7942 and ten colonies of strain GDEFE7942 were randomly picked from plates spread with diluted day-13 cultures shown in b-d and were 
subjected to colony PCR using primers flanking the efe-insertion site on the genome. Red arrows indicate the expected PCR product size for strains EFE7942 and GD-EFE7942; black arrows indicate the expected PCR product size for the wild-type 7942 strain. $g$, DNA sequencing of the PCR products obtained in $f$ revealed mutations of the EFE expression cassettes on the genomes of all ten randomly picked EFE7942 colonies, whereas no mutations arose within the genomic region of the EFE expression cassette in any of the ten GDEFE7942 colonies.

\section{Supplementary Files}

This is a list of supplementary files associated with this preprint. Click to download.

- SupplementaryData1Arginasesunderthecontrolofguanidineriboswitches.xlsx

- GuanidinedegradationSInc1stsubmission.docx

- SupplementaryData2AlignmentofProteinSequenceofSII1077fromSynechocystisspPCC6803top1000.pdf 